\title{
Time-dependent chronoamperometric response of dual inlaid disk electrodes
}

\author{
Christopher G. Bell ${ }^{\mathrm{a}, *}$, Peter D. Howell ${ }^{\mathrm{a}}$, Howard A. Stone ${ }^{\mathrm{b}}$ \\ ${ }^{a}$ Mathematical Institute, University of Oxford, 24-29 St Giles', Oxford, OX1 3LB, UK \\ ${ }^{b}$ Department of Mechanical and Aerospace Engineering, Princeton University, Princeton, New Jersey \\ 08544, United States
}

\begin{abstract}
Dual-disk electrodes allow for novel electrochemical investigations, particularly when used in generator-collector mode. However analytical understanding of how the current response of each individual electrode is affected by the presence of the neighbouring electrode is limited. In this article, we derive an approximate analytical solution for the time-dependent chronoamperometric current response of a pair of electrodes, each subjected to a different initial potential step. The solution is valid when the centre-to-centre distance between the disks is much larger than the disk radii, and for times such that the diffusion layers generated at each electrode have started to interact. It accommodates quasi-reversible reactions and unequal diffusion coefficients of the oxidant and the reductant. The generator-collector system is a specific case, and we deduce simple expressions for the time-dependent current and steady-state collection efficiencies at each electrode. The analytical solution facilitates analysis of shielding and feedback effects, and shows the explicit dependence of the currents on the underlying electrochemical parameters.
\end{abstract}

Keywords: chronoamperometry, dual-disk, dual-electrode, collector, generator, microdisk

\section{Introduction}

The use of dual-electrode systems extends the range of investigative techniques available for electrochemical analysis. A particularly important application is the generator-collector mode, whereby a species is oxidised/reduced at one electrode, and the product is subsequently reduced/oxidised at the neighbouring electrode. A comprehensive review of the many dual-electrode geometries and their use as generator-collector systems can be found in the article by Barnes et al. [1]. They discuss rotating ring-disks, wall-jet ring-disks and double channel flow electrodes, which exploit forced convection to transport matter between the electrodes, and other systems, such as parallel planar

\footnotetext{
${ }^{*}$ Corresponding author

Email address: bell@maths.ox.ac.uk (Christopher G. Bell)
} 
microbands, dual disks, dual hemispheres, and ring-recessed disks, which use only diffusion. They also report many applications, including investigation into the lifetimes of electro-generated species, detection and characterisation of intermediate species in electrochemical reactions, detection of sub-micromolar concentrations of analyte, and many more.

In this article, we consider the general time-dependent behaviour (including the generator-collector mode) of dual inlaid disk electrode systems in which diffusion is the only means of mass transport to the electrodes. The system consists of a pair of neighbouring microdisk electrodes inlaid into an insulating substrate, and retains the practical advantages of the single inlaid microdisk electrode, namely that it is simple to fabricate and the distorting influence of ohmic drop and double-layer capacitance on the Faradaic current is greatly reduced [2-4]. Microdisk electrodes also enable accurate electrochemical investigations into reactions with fast kinetics, which would be impossible to resolve at larger electrodes [5]. Dual-disk electrode systems have been used in the literature to improve selectivity in electrochemical detection [6], and to perform simultaneous oxidative and reductive detection [7]. However, analysis of the current response of a dual-disk system is complicated by the effects of cross-talk between the electrodes. Figure 1 shows a cartoon of the evolution of the diffusion layers over time at a dual-disk system where the potentials at each electrode have been set such that the same species is being oxidised/reduced simultaneously at both electrodes (i.e. not in generator-collector mode). At very short times, the diffusion layer behaves as if the diffusion was planar at each electrode as shown in Figure 1 (a); then at intermediate times as shown in Figure 1 (b), the electrodes remain independent, but the diffusion layer at each electrode has grown to be three-dimensional; and finally the electrodes cease to be independent over time-scales such that the diffusion layers have begun to overlap, as shown in Figure 1 (c). Competition between the diffusion layers at this stage causes a reduction in the total current response of a dual-disk system, when compared with the sum of the currents through two independent single disks. This reduction in the current response is known as shielding. For a dual electrode system in generator-collector mode, the opposite occurs. The current at the generator electrode is enhanced due to the overlap of the diffusion layers, since the reverse reaction at the collector electrode adds to the diffusion layer around the generator electrode; this effect is known as feedback. Zhang and Zhou [8] and Matysik [9] fabricated platinum dualdisk electrodes and investigated the shielding and feedback effects. Baur et al. [10] also investigated the diffusional interaction in a dual-microdisk system.

Whilst dual-disk systems undoubtedly have many useful applications, theoretical understanding of the current response, and in particular shielding and feedback effects, remains limited. The boundary element method has been used to simulate numerically the steady-state (Fulian et al. [11]) and transient (Qiu and Fisher [12]) current response of a generator-collector system under diffusion control. Baur and Motsegood [13] investigated the time-dependent chronoamperometric response of dual-disk electrodes under diffusion control using three-dimensional random walk simulations. Recently, Cutress et al. [14] used the explicit finite difference method to simulate transient current responses for generator-collector systems. Analytical solutions for dual-disk systems in the literature include that by Strieder [15], which is based on investigations in potential theory reported in Sneddon [16]. Strieder's solution is limited to the steady- 
state current when both electrodes are held at potentials in the diffusion-limited regime, and allows the shielding effect to be calculated. The analysis assumes that the redox species have equal diffusion coefficients and that the disks have equal radii. Phillips and Stone [17] performed theoretical investigations into the steady-state current response of collector-generator systems with and without a homogeneous first-order reaction, and in particular derived an approximate theoretical solution for the steady-state collection efficiency of a dual-disk system; the higher-order correction terms in their solution require the assumption that the current density on the generator electrode surface is uniform.

In Section 2 of this article, we use the method of matched asymptotic expansions to derive an analytical solution for the long-time-dependent chronoamperometric current through two neighbouring disk electrodes inlaid into an insulating surface. The electrodes are subjected to arbitrary initial potential steps, and we assume that the redox reaction at each electrode is quasi-reversible. The diffusion coefficients of the oxidant and reductant, $\tilde{D}_{O}$ and $\tilde{D}_{R}\left(\mathrm{~m} \mathrm{~s}^{-2}\right)$, are allowed to be unequal. The solution for the current response requires that the ratio of the radii $\tilde{a}_{1,2}(\mathrm{~m})$ of the electrodes to the distance $\tilde{L}(\mathrm{~m})$ separating their centres is small, i.e.

$$
\frac{\tilde{a}_{j}}{\tilde{L}} \ll 1, \quad j=1,2,
$$

and it is valid for long times, $\tilde{t}$, when the electrodes have ceased to behave individually and their diffusion layers have begun to overlap, as depicted in Figure 1 (c). This occurs over time-scales of the following order:

$$
\tilde{t}=O\left(\max \left\{\frac{\tilde{L}^{2}}{\tilde{D}_{O}}, \frac{\tilde{L}^{2}}{\tilde{D}_{R}}\right\}\right),
$$

which corresponds to diffusion-layer thicknesses of $O(\tilde{L})$.

For the reader less interested in the mathematical details, the theoretical problem is presented schematically in Figure 2, and the final analytical result for the approximate long-time-dependent quasi-reversible chronoamperometric current through disk 1 is presented in expression (60). The equivalent expression for the current through disk 2 can be obtained by interchanging the subscripts 1 and 2 in (60). The analytical solution allows for explicit quantification of the effects of shielding and feedback in terms of the parameters of the electrochemical system.

In Section 3, we discuss the implications of our analytical solution, in particular for the important special cases listed below, along with equation numbers for easy reference:

- the quasi-reversible steady-state current response (62);

- the time-dependent and steady-state (63) current responses for reversible reactions;

- the diffusion-limited current responses due to extreme polarisation of both electrodes: for a reduction reaction, the time-dependent and steady-state responses 
are given by (64) and (65) respectively, and for an oxidation reaction by (67) and (68);

- the time-dependent current responses of generator-collector systems: for a reduction reaction at the generator, the currents at the generator and collector are given by (69) and (70) respectively, and, for an oxidation reaction at the generator, they are given by (71) and (72). A formula for the steady-state collection efficiency in both cases is presented in expression (73).

For the diffusion-limited currents due to extreme polarisation, we show that our solution encompasses the steady-state diffusion-limited solution of Strieder [15] for disks of identical radii. We also compare our expression for the steady-state collection efficiency of a generator-collector system to the expression derived by Phillips and Stone [17] and to a formula fitted to numerically simulated results for disks of identical radii derived by Cutress et al. [14]. Finally, we use our solution to illustrate the effects of shielding and feedback, and demonstrate that unequal diffusion coefficients of the oxidant and the reductant can have a large impact on the current response.

\section{Theory}

\subsection{Governing equations and non-dimensionalisation}

We consider a simple redox reaction between two species, Ox and Red, diffusing in the half-space $\tilde{z}>0$ (tildes indicate dimensional variables), above two disk electrodes inlaid into the plane $\tilde{z}=0$ with with radii $\tilde{a}_{j}(j=1,2)$ and centres at $(-\tilde{L} / 2,0,0)$ and $(+\tilde{L} / 2,0,0)$ in the cartesian coordinate system $(\tilde{x}, \tilde{y}, \tilde{z})$. It is convenient to define also two polar coordinate systems $\left(\tilde{r}_{j}, \tilde{z}, \theta_{j}\right)$ with origin at the centre of disk $j(j=1,2)$, and the angles $\theta_{j}$ are measured from the line joining the centres of the disks, as shown in the schematic of the theoretical problem depicted in Figure 2. To make progress with this problem, we will assume that $\tilde{a}_{j} / \tilde{L} \ll 1$, so that the distance between the centres of the disks is very much greater than the radii of the disks, and we will look for a solution for the long-time transient current response, when the electrode diffusion layers begin to overlap and the reaction at each electrode starts to influence the reaction at the other electrode, i.e. the solution will be valid for time-scales of the order detailed in expression (2).

We assume that a redox reaction takes place at each electrode, in which $n$ electrons are exchanged:

$$
\mathrm{Ox}+n \mathrm{e} \underset{\tilde{k}_{b, j}}{\stackrel{\tilde{k}_{f, j}}{\rightleftharpoons}} \mathrm{Red} .
$$

Here $\tilde{k}_{f, j}$ and $\tilde{k}_{b, j}$ are the forward and backward rate constants for the reaction, which can be different at each electrode $j$. We will however assume that the electrodes are held at a constant potential so that $\tilde{k}_{f, j}$ and $\tilde{k}_{b, j}$ do not vary with time.

REMOVED FIRST SENTENCE If we neglect any effects due to migration and convection, then the concentrations of Ox and $\operatorname{Red}, \tilde{C}_{O}(\tilde{x}, \tilde{y}, \tilde{z}, \tilde{t})$ and $\tilde{C}_{R}(\tilde{x}, \tilde{y}, \tilde{z}, \tilde{t})$, 
satisfy the unsteady diffusion equation for $\tilde{z}>0$ with constant diffusion coefficients $\tilde{D}_{O}$ and $\tilde{D}_{R}\left(\mathrm{~m}^{2} \mathrm{~s}^{-1}\right)$. Hence

$$
\tilde{D}_{O} \nabla^{2} \tilde{C}_{O}=\frac{\partial \tilde{C}_{O}}{\partial \tilde{t}}, \quad \tilde{D}_{R} \nabla^{2} \tilde{C}_{R}=\frac{\partial \tilde{C}_{R}}{\partial \tilde{t}}, \quad \text { for } \tilde{z}>0 .
$$

Initially the bulk concentrations of each species are constant everywhere:

$$
\tilde{C}_{O}(\tilde{x}, \tilde{y}, \tilde{z}, 0)=\tilde{C}_{O}^{*}, \quad \tilde{C}_{R}(\tilde{x}, \tilde{y}, \tilde{z}, 0)=\tilde{C}_{R}^{*}, \quad \text { at } \tilde{t}=0 .
$$

We assume that the bulk concentrations far away from the electrodes remain undisturbed as the reaction progresses, so that the far-field boundary conditions are

$$
\tilde{C}_{O} \rightarrow \tilde{C}_{O}^{*}, \quad \tilde{C}_{R} \rightarrow \tilde{C}_{R}^{*}, \quad \text { as } \tilde{z} \rightarrow \infty
$$

On each electrode surface, the boundary conditions are given by the reaction at the surface and conservation of matter:

$$
\left.\begin{array}{l}
\tilde{D}_{O} \frac{\partial \tilde{C}_{O}}{\partial \tilde{z}}=\tilde{k}_{f, j} \tilde{C}_{O}-\tilde{k}_{b, j} \tilde{C}_{R}, \\
\tilde{D}_{O} \frac{\partial \tilde{C}_{O}}{\partial \tilde{z}}=-\tilde{D}_{R} \frac{\partial \tilde{C}_{R}}{\partial \tilde{z}},
\end{array}\right\} \quad \text { for } \tilde{r}_{j} \leq \tilde{a}_{j}, \tilde{z}=0
$$

There is no flux through the remainder of the surface, so that

$$
\tilde{D}_{O} \frac{\partial \tilde{C}_{O}}{\partial \tilde{z}}=\tilde{D}_{R} \frac{\partial \tilde{C}_{R}}{\partial \tilde{z}}=0, \quad \text { for } \tilde{r}_{j}>\tilde{a}_{j}, \tilde{z}=0 .
$$

The Faradaic current through electrode $j$ is given by

$$
\tilde{I}_{j}(\tilde{t})=-n \tilde{F} \tilde{D}_{O} \int_{0}^{2 \pi} \int_{0}^{\tilde{a}_{j}} \frac{\partial \tilde{C}_{O}}{\partial \tilde{z}}\left(\tilde{r}_{j}, 0, \theta_{j}, \tilde{t}\right) \tilde{r}_{j} d \tilde{r}_{j} d \theta_{j},
$$

where $\tilde{F}$ is Faraday's constant and $n$ is the number of electrons transferred in the redox reaction.

We choose the following scalings to non-dimensionalise the problem:

$$
\begin{gathered}
\tilde{x}=\tilde{a} x, \quad \tilde{y}=\tilde{a} y, \quad \tilde{z}=\tilde{a} z, \quad \tilde{t}=\frac{\tilde{L}^{2}}{\tilde{D}} t, \\
\tilde{a}_{j}=\tilde{a} a_{j}, \quad \tilde{D}_{O}=\tilde{D} D_{O}, \quad \tilde{D}_{R}=\tilde{D} D_{R}, \\
\tilde{C}_{O}=\tilde{C}_{O}^{*}-\tilde{C}^{*} C_{O}, \quad \tilde{C}_{R}=\tilde{C}_{R}^{*}-\tilde{C}^{*} C_{R}, \quad \tilde{I}_{j}=n \tilde{F} \tilde{D}_{O} \tilde{C}^{*} \tilde{a} I_{j} .
\end{gathered}
$$

Here $\tilde{a}$ is the typical size of the radii of the disks $\tilde{a}_{j}$ and $\tilde{D}$ is the typical size of the diffusion coefficients $\tilde{D}_{O}$ and $\tilde{D}_{R}$. We have chosen the time-scale to be $\tilde{L}^{2} / \tilde{D}$, since this represents the time-scale for the species to diffuse from one disk to the other, and hence is the time-scale over which the diffusion layers of the electrodes start to interact; the solution derived below is only valid over time-scales of this order. On time-scales 
of order less than this, the two disks remain independent. The concentration scaling $\tilde{C}^{*}$ is related to the typical size of the bulk concentrations $\tilde{C}_{O}^{*}$ and $\tilde{C}_{R}^{*}$.

When we define $\epsilon$ to be the ratio of the typical electrode radius to the distance separating their centres:

$$
\epsilon=\frac{\tilde{a}}{\tilde{L}} \ll 1,
$$

the problem (4) can be written in terms of the non-dimensional variables as:

$$
D_{O} \nabla^{2} C_{O}=\epsilon^{2} \frac{\partial C_{O}}{\partial t}, \quad D_{R} \nabla^{2} C_{R}=\epsilon^{2} \frac{\partial C_{R}}{\partial t}, \quad \text { for } z>0,
$$

with initial conditions:

$$
C_{O}(x, y, z, 0)=0, \quad C_{R}(x, y, z, 0)=0, \quad \text { at } t=0,
$$

and boundary conditions:

$$
\begin{aligned}
& C_{O} \rightarrow 0, \quad C_{R} \rightarrow 0, \quad \text { as } z \rightarrow \infty, \\
& \left.\begin{array}{rl}
-\frac{\partial C_{O}}{\partial z} & =k_{f, j} C_{O}^{*}-k_{b, j} C_{R}^{*}-k_{f, j} C_{O}+k_{b, j} C_{R}, \\
D_{O} \frac{\partial C_{O}}{\partial z} & =-D_{R} \frac{\partial C_{R}}{\partial z},
\end{array}\right\} \quad \text { for } r_{j} \leq a_{j}, z=0, \\
& \frac{\partial C_{O}}{\partial z}=\frac{\partial C_{R}}{\partial z}=0, \quad \text { for } r_{j}>a_{j}, z=0 .
\end{aligned}
$$

Here $C_{O}^{*}=\tilde{C}_{O}^{*} / \tilde{C}^{*}$ and $C_{R}^{*}=\tilde{C}_{R}^{*} / \tilde{C}^{*}$, and the non-dimensional rate constants are given by:

$$
k_{f, j}=\frac{\tilde{k}_{f, j} \tilde{a}}{\tilde{D}_{O}}, \quad k_{b, j}=\frac{\tilde{k}_{b, j} \tilde{a}}{\tilde{D}_{O}} .
$$

The non-dimensional Faradaic current through the electrode $j$ is calculated from:

$$
I_{j}(t)=\int_{0}^{2 \pi} \int_{0}^{a_{j}} \frac{\partial C_{O}}{\partial z}\left(r_{j}, 0, \theta_{j}, t\right) r_{j} d r_{j} d \theta_{j} .
$$

\subsection{Asymptotic solution for the current at each electrode}

Since $\epsilon \ll 1$, there is an inner region near each disk where the concentrations are at steady-state to leading order, and an outer region where the concentrations are timedependent to leading order. We will find the solutions in both regions using matched asymptotics in a similar manner to the single-disk problem descibed in our previous article [18]. In the inner region near disk $j$, the coordinate system is $\left(r_{j}, z, \theta_{j}\right)$ as defined above, and variables will be $C_{O, j}\left(r_{j}, z, \theta_{j}, t\right)$ and $C_{R, j}\left(r_{j}, z, \theta_{j}, t\right)$. In the outer region, we will define the coordinate system in terms of 'hatted' variables, $(\hat{x}, \hat{y}, \hat{z})$, where $\hat{x}=\epsilon x, \hat{y}=\epsilon y$ and $\hat{z}=\epsilon z$. In terms of the polar coordinate systems centred at each disk $j$, the outer coordinate system can be written $\left(\hat{r}_{j}, \hat{z}, \theta_{j}\right)$, where $\hat{r}_{j}=\epsilon r_{j}$. Similarly the outer dependent variables will also have hats, i.e. $\hat{C}_{O}(\hat{x}, \hat{y}, \hat{z}, t)$ and $\hat{C}_{R}(\hat{x}, \hat{y}, \hat{z}, t)$. 
We expand the inner dependent variables in the following perturbation series:

$$
\begin{aligned}
& C_{O, j}=C_{O, j}^{(0)}+\epsilon C_{O, j}^{(1)}+\epsilon^{2} C_{O, j}^{(2)}+O\left(\epsilon^{3}\right), \\
& C_{R, j}=C_{R, i}^{(0)}+\epsilon C_{R, j}^{(1)}+\epsilon^{2} C_{R, j}^{(2)}+O\left(\epsilon^{3}\right),
\end{aligned}
$$

and similarly for the outer variables:

$$
\begin{aligned}
& \hat{C}_{O}=\epsilon \hat{C}_{O}^{(0)}+\epsilon^{2} \hat{C}_{O}^{(1)}+O\left(\epsilon^{3}\right), \\
& \hat{C}_{R}=\epsilon \hat{C}_{R}^{(0)}+\epsilon^{2} \hat{C}_{R}^{(1)}+O\left(\epsilon^{3}\right) .
\end{aligned}
$$

We remark in advance that construction of the asymptotic solution reveals that the inner solutions are axi-symmetric up to $O(\epsilon)$, and the outer solutions are radially symmetric up to $O\left(\epsilon^{2}\right)$.

\subsubsection{Leading-order inner solution}

To leading order the inner solution in the vicinity of each disk is axisymmetric and steady state, so that $C_{O, j}^{(0)}\left(r_{j}, z\right)$ and $C_{R, j}^{(0)}\left(r_{j}, z\right)$ satisfy the following steady problem:

$$
\nabla^{2} C_{O, j}^{(0)}=0, \quad \nabla^{2} C_{R, j}^{(0)}=0, \quad \text { for } z>0,
$$

with boundary conditions $(8 \mathrm{c})$ to $(8 \mathrm{e})$. Since the following quantity is conserved:

$$
D_{O} C_{O, j}^{(0)}+D_{R} C_{R, j}^{(0)}=0
$$

$C_{R, j}^{(0)}$ can be eliminated to obtain a problem for the single variable $C_{O, j}^{(0)}$ :

$$
\begin{array}{rlrl}
\nabla^{2} C_{O, j}^{(0)} & =0, & & z>0, \\
C_{O, j}^{(0)} & \rightarrow 0, & & z \rightarrow \infty, \\
\frac{\partial C_{O, j}^{(0)}}{\partial z} & = \begin{cases}0 & r_{j}>a_{j},\end{cases} \\
\beta_{j} C_{O, j}^{(0)}-\alpha_{j} & r_{j} \leq a_{j}, & z=0,
\end{array}
$$

where

$$
\begin{aligned}
\alpha_{j} & =k_{f, j} C_{O}^{*}-k_{b, j} C_{R}^{*}, \\
\beta_{j} & =k_{f, j}+k_{b, j} \frac{D_{O}}{D_{R}} .
\end{aligned}
$$

Therefore the solutions for $C_{O, i}^{(0)}$ and $C_{R, i}^{(0)}$ can be written as:

$$
\begin{aligned}
C_{O, j}^{(0)}(r, z) & =\frac{\alpha_{j}}{\beta_{j}} \chi\left(a_{j} \beta_{j} ; \frac{r_{j}}{a_{j}}, \frac{z}{a_{j}}\right), \\
C_{R, j}^{(0)}(r, z) & =-\frac{D_{O} \alpha_{j}}{D_{R} \beta_{j}} \chi\left(a_{j} \beta_{j} ; \frac{r_{j}}{a_{j}}, \frac{z}{a_{j}}\right),
\end{aligned}
$$


where $\chi(\beta ; r, z)$ satisfies the following problem:

$$
\begin{aligned}
& \nabla^{2} \chi=0, \quad z>0, \\
& \chi \rightarrow 0, \quad z \rightarrow \infty, \\
& \frac{\partial \chi}{\partial z}=q(\beta ; r)=\left\{\begin{array}{ll}
0 & r>1, \\
\beta(\chi-1) & r \leq 1,
\end{array} \quad z=0 .\right.
\end{aligned}
$$

As in [18-20], the solution for $\chi(\beta ; r, z)$ can be written in terms of the unknown flux through the boundary, $q(\beta ; r)$, as:

$$
\chi(\beta ; r, z)=-\frac{2}{\pi} \int_{0}^{1} \mathrm{~K}\left(\frac{4 r s}{(r+s)^{2}+z^{2}}\right) \frac{q(\beta ; s) s \mathrm{~d} s}{\sqrt{(r+s)^{2}+z^{2}}},
$$

where $\mathrm{K}(\cdot)$ denotes a complete elliptic integral of the first kind ([21], p.590, 17.3.1):

$$
\mathrm{K}(m)=\int_{0}^{\frac{\pi}{2}} \frac{1}{\sqrt{1-m \sin ^{2} \theta}} \mathrm{d} \theta .
$$

This enables us to write the far-field behaviour of $\chi(\beta ; r, z)$ as:

$$
\chi(\beta ; r, z) \sim-\frac{I_{s s}(\beta)}{2 \pi \sqrt{r^{2}+z^{2}}}-\frac{\left(r^{2}-2 z^{2}\right)}{4\left(r^{2}+z^{2}\right)^{5 / 2}} \int_{0}^{1} q(\beta ; s) s^{3} \mathrm{~d} s+\cdots \quad \text { as } r^{2}+z^{2} \rightarrow \infty,
$$

which is important for matching to the outer solution. Here $I_{s s}(\beta)$ is given by:

$$
I_{s s}(\beta)=2 \pi \int_{0}^{1} q(\beta ; r) r \mathrm{~d} r=2 \pi \int_{0}^{1} \frac{\partial \chi}{\partial z}(\beta ; r, 0) r \mathrm{~d} r
$$

and it can be calculated using Tranter's method [22] to solve (19) in terms of a series of Bessel functions, as described in our previous article [18]; in that article, we also included in the Supplementary Data a working curve for $I_{s s}(\beta)$ for $\beta$ in the range $0 \leq \beta \leq 500$. For values of $\beta>500, I_{s s}(\beta)$ can be calculated using the asymptotic expansion derived by Phillips [23] for large $\beta$ :

$$
I_{s s}(\beta)=-4\left(1-(\pi \beta)^{-1} \log \beta+o\left(\beta^{-1} \log \beta\right)\right), \quad \text { as } \beta \rightarrow \infty .
$$

\subsubsection{Leading-order outer solution}

We use Van Dyke's method [24] to find the leading-order impact in the far field of the leading-order inner solutions at the two disks. Writing the inner solutions $C_{O, j}^{(0)}$ and $C_{R, j}^{(0)}(18)$ in terms of the outer variables $\hat{r}_{j}$ and $\hat{z}$, using the far-field behaviour of $\chi(\beta ; r, z)$ given by (22), and letting $\epsilon$ tend to zero, we find that

$$
\begin{aligned}
& C_{O, j}^{(0)}\left(\hat{r}_{j}, \hat{z}\right)=-\epsilon \frac{\alpha_{j} a_{j}}{\beta_{j}} \frac{I_{s s}\left(a_{j} \beta_{j}\right)}{2 \pi \hat{\rho}_{j}}+O\left(\epsilon^{3}\right), \\
& C_{R, j}^{(0)}\left(\hat{r}_{j}, \hat{z}\right)=\epsilon \frac{D_{O} \alpha_{j} a_{j}}{D_{R} \beta_{j}} \frac{I_{s s}\left(a_{j} \beta_{j}\right)}{2 \pi \hat{\rho}_{j}}+O\left(\epsilon^{3}\right),
\end{aligned}
$$


where $\hat{\rho}_{j}=\sqrt{\hat{r}_{j}^{2}+\hat{z}^{2}}$, and these expressions give the matching conditions for the outer solutions as $\hat{\rho}_{j} \rightarrow 0$. Then the leading-order outer solutions, $\hat{C}_{O}^{(0)}$ and $\hat{C}_{R}^{(0)}$, are the sum of contributions from each of the disks:

$$
\hat{C}_{O}^{(0)}=\sum_{j=1}^{2} \hat{C}_{O, j}^{(0)}\left(\hat{\rho}_{j}, t\right), \quad \hat{C}_{R}^{(0)}=\sum_{j=1}^{2} \hat{C}_{R, j}^{(0)}\left(\hat{\rho}_{j}, t\right),
$$

where the functions $\hat{C}_{O, j}^{(0)}\left(\hat{\rho}_{j}, t\right)$ and $\hat{C}_{R, j}^{(0)}\left(\hat{\rho}_{j}, t\right)$ satisfy the following problems:

$$
\begin{array}{rlrl}
\frac{D}{\hat{\rho}_{j}} \frac{\partial^{2}}{\partial \hat{\rho}_{j}^{2}}\left(\hat{\rho}_{j} \hat{C}_{O, j}^{(0)}\right) & =\frac{\partial \hat{C}_{O, j}^{(0)}}{\partial t}, & z>0, \\
\hat{C}_{O, j}^{(0)} & =0, & t & =0, \\
\hat{C}_{O, j}^{(0)} & \rightarrow 0, & \hat{\rho}_{j} \rightarrow \infty \\
\hat{C}_{O, j}^{(0)} \sim-\frac{\alpha_{j} a_{j}}{\beta_{j}} \frac{I_{s s}\left(a_{j} \beta_{j}\right)}{2 \pi \hat{\rho}_{j}}, & \hat{\rho}_{j} \rightarrow 0
\end{array}
$$

and

$$
\begin{array}{rlrl}
\frac{D_{R}}{\hat{\rho}_{j}} \frac{\partial^{2}}{\partial \hat{\rho}_{j}^{2}}\left(\hat{\rho}_{j} \hat{C}_{R, j}^{(0)}\right) & =\frac{\partial \hat{C}_{R, j}^{(0)}}{\partial t}, & z>0, \\
\hat{C}_{R, j}^{(0)} & =0, & t & =0, \\
\hat{C}_{R, j}^{(0)} & \rightarrow 0, & \hat{\rho}_{j} \rightarrow \infty, \\
\hat{C}_{R, j}^{(0)} & \sim \frac{D_{O} \alpha_{j} a_{j}}{D_{R} \beta_{j}} \frac{I_{s s}\left(a_{j} \beta_{j}\right)}{2 \pi \hat{\rho}_{j}}, & \hat{\rho}_{j} \rightarrow 0 .
\end{array}
$$

The solutions to these problems are:

$$
\begin{aligned}
\hat{C}_{O, j}^{(0)}\left(\hat{\rho}_{j}, t\right) & =-\frac{\alpha_{j} a_{j}}{\beta_{j}} \frac{I_{s s}\left(a_{j} \beta_{j}\right)}{2 \pi \hat{\rho}_{j}} \operatorname{erfc}\left(\frac{\hat{\rho}_{j}}{2 \sqrt{D_{O} t}}\right), \\
\hat{C}_{R, j}^{(0)}\left(\hat{\rho}_{j}, t\right) & =\frac{D_{O}}{D_{R}} \frac{\alpha_{j} a_{j}}{\beta_{j}} \frac{I_{s s}\left(a_{j} \beta_{j}\right)}{2 \pi \hat{\rho}_{j}} \operatorname{erfc}\left(\frac{\hat{\rho}_{j}}{2 \sqrt{D_{R} t}}\right) .
\end{aligned}
$$

\subsubsection{First-order inner solution near each disk}

To apply Van Dyke's rule again, let us consider the two-term inner expansion of the one-term outer solutions (26) near disk 1 . We note that $\hat{\rho}_{1}=\epsilon \rho_{1}$, where $\rho_{1}=\sqrt{r_{1}^{2}+z^{2}}$, and $\hat{\rho}_{2}$ can be written in terms of the inner variables $\left(r_{1}, z, \theta_{1}\right)$ as:

$$
\hat{\rho}_{2}=\left(1-2 \epsilon r_{1} \cos \theta_{1}+\epsilon^{2}\left(r_{1}^{2}+z^{2}\right)\right)^{\frac{1}{2}} .
$$

Therefore, substituting expressions (29) for $\hat{C}_{O, j}^{(0)}\left(\hat{\rho}_{j}, t\right)$ and $\hat{C}_{R, j}^{(0)}\left(\hat{\rho}_{j}, t\right)$ into (26), writing (26) in terms of the inner variables, letting $\epsilon \rightarrow 0$ and finding the two-term inner expansion near disk 1 , we can deduce the boundary conditions as $\rho_{1} \rightarrow \infty$ for 
the first-order inner problem near disk 1 . This results in the following boundary value problem for $C_{O, 1}^{(1)}$ and $C_{R, 1}^{(1)}$ :

$$
\begin{gathered}
\nabla^{2} C_{O, 1}^{(1)}=0, \quad \nabla^{2} C_{R, 1}^{(1)}=0, \quad \text { for } z>0, \\
-\frac{\partial C_{O, 1}^{(1)}}{\partial z}=-k_{f, 1} C_{O, 1}^{(1)}+k_{b, 1} C_{R, 1}^{(1)}, \\
-\frac{\partial C_{O, 1}^{(1)}}{\partial z}=\frac{D_{R}}{D_{O}} \frac{\partial C_{R, 1}^{(1)}}{\partial z}, \\
\frac{\partial C_{O, 1}^{(1)}}{\partial z}=\frac{\partial C_{R, 1}^{(1)}}{\partial z}=0, \quad \text { for } z=0, r_{1}>a_{1}, \\
\text { for } z=0, r_{1} \leq a_{1}, \\
C_{O, 1}^{(1)} \sim C_{O, 1}^{(1), \infty}(t), \quad C_{R, 1}^{(1)} \sim-\frac{D_{O}}{D_{R}} C_{R, 1}^{(1), \infty}(t), \quad \text { as } \rho_{1} \rightarrow \infty
\end{gathered}
$$

where

$$
\begin{aligned}
& C_{O, 1}^{(1), \infty}(t)=\frac{\alpha_{1} a_{1} I_{s s}\left(a_{1} \beta_{1}\right)}{2 \pi^{\frac{3}{2}} \beta_{1} \sqrt{D_{O} t}}-\frac{\alpha_{2} a_{2} I_{s s}\left(a_{2} \beta_{2}\right)}{2 \pi \beta_{2}} \operatorname{erfc}\left(\frac{1}{2 \sqrt{D_{O} t}}\right) \\
& C_{R, 1}^{(1), \infty}(t)=\frac{\alpha_{1} a_{1} I_{s s}\left(a_{1} \beta_{1}\right)}{2 \pi^{\frac{3}{2}} \beta_{1} \sqrt{D_{R} t}}-\frac{\alpha_{2} a_{2} I_{s s}\left(a_{2} \beta_{2}\right)}{2 \pi \beta_{2}} \operatorname{erfc}\left(\frac{1}{2 \sqrt{D_{R} t}}\right)
\end{aligned}
$$

Since it can be deduced from (31) that

$$
C_{O, 1}^{(1)}+\frac{D_{R}}{D_{O}} C_{R, 1}^{(1)}=C_{O, 1}^{(1), \infty}(t)-C_{R, 1}^{(1), \infty}(t)
$$

it follows that $C_{R, 1}^{(1)}$ can be eliminated from (31) to obtain a single problem for $C_{O, 1}^{(1)}$ :

$$
\begin{aligned}
\nabla^{2} C_{O, 1}^{(1)} & =0, & & z>0, \\
C_{O, 1}^{(1)} & \rightarrow C_{O, 1}^{(1), \infty}(t), & \rho_{1} \rightarrow \infty, & \\
\frac{\partial C_{O, 1}^{(1)}}{\partial z} & =\beta_{1} C_{O, 1}^{(1)}-\frac{k_{b, 1} D_{O}}{D_{R}}\left(C_{O, 1}^{(1), \infty}(t)-C_{R, 1}^{(1), \infty}(t)\right), & & r_{1}<a_{1}, z=0 \\
\frac{\partial C_{O, 1}^{(1)}}{\partial z} & =0, & & r_{1}>a_{1}, z=0 .
\end{aligned}
$$

This can be written in similar form to (19), so that the solutions for $C_{O, 1}^{(1)}$ and $C_{R, 1}^{(1)}$ are given by:

$$
\begin{aligned}
C_{O, 1}^{(1)}\left(r_{1}, z, t\right)= & C_{O, 1}^{(1), \infty}(t)- \\
& \left(\frac{k_{f, 1} C_{O, 1}^{(1), \infty}(t)+\frac{k_{b, 1} D_{O}}{D_{R}} C_{R, 1}^{(1), \infty}(t)}{\beta_{1}}\right) \chi\left(a_{1} \beta_{1} ; \frac{r_{1}}{a_{1}}, \frac{z}{a_{1}}\right),
\end{aligned}
$$




$$
\begin{aligned}
C_{R, 1}^{(1)}\left(r_{1}, z, t\right) & =-\frac{D_{O}}{D_{R}} C_{R, 1}^{(1), \infty}(t)+ \\
\frac{D_{O}}{D_{R}} & \left(\frac{k_{f, 1} C_{O, 1}^{(1), \infty}(t)+\frac{k_{b, 1} D_{O}}{D_{R}} C_{R, 1}^{(1), \infty}(t)}{\beta_{1}}\right) \chi\left(a_{1} \beta_{1} ; \frac{r_{1}}{a_{1}}, \frac{z}{a_{1}}\right) .
\end{aligned}
$$

We note that the first-order solutions in the vicinity of the second disk, $C_{O, 2}^{(1)}$ and $C_{R, 2}^{(1)}$, are found by symmetry by interchanging the subscripts 1 and 2 in (35).

\subsubsection{First-order outer solution}

The first-order terms of the outer expansion, $\hat{C}_{O}^{(1)}$ and $\hat{C}_{R}^{(1)}$, contain contributions from the outer expansions of the inner expansions near disk 1 and disk 2 , and therefore have the following forms:

$$
\hat{C}_{O}^{(1)}=\sum_{j=1}^{2} \hat{C}_{O, j}^{(1)}\left(\hat{\rho}_{j}, t\right), \quad \hat{C}_{R}^{(1)}=\sum_{j=1}^{2} \hat{C}_{R, j}^{(1)}\left(\hat{\rho}_{j}, t\right) .
$$

Further application of Van Dyke's rule allows us to obtain these first-order terms. Let us consider the two-term outer expansion of the two-term inner expansion for the oxidant near disk 1, i.e. the outer expansion of $C_{O, 1}^{(0)}+\epsilon C_{O, 1}^{(1)}$, where $C_{O, 1}^{(0)}$ is given by (18a) with $j=1$, and $C_{O, 1}^{(1)}$ is given by (35a). This gives the matching condition for $\hat{C}_{O, 1}^{(1)}\left(\hat{\rho}_{1}, t\right)$ as $\hat{\rho}_{1} \rightarrow 0$, and hence $\hat{C}_{O, 1}^{(1)}\left(\hat{\rho}_{1}, t\right)$ must satisfy:

$$
\begin{aligned}
\frac{D_{O}}{\hat{\rho}_{1}} \frac{\partial^{2}}{\partial \hat{\rho}_{1}^{2}}\left(\hat{\rho}_{1} \hat{C}_{O, 1}^{(1)}\right) & =\frac{\partial \hat{C}_{O, 1}^{(1)}}{\partial t}, & \text { for } \hat{z}>0, \\
\hat{C}_{O, 1}^{(1)} & =0, & \text { at } t=0, \\
\hat{C}_{O, 1}^{(1)} & \sim \hat{C}_{O, 1}^{(1), 0}\left(\hat{\rho}_{1}, t\right), & \text { as } \hat{\rho}_{1} \rightarrow 0, \\
\hat{C}_{O, 1}^{(1)} & \rightarrow 0, & \text { as } \hat{\rho}_{1} \rightarrow \infty,
\end{aligned}
$$

where

$$
\begin{gathered}
\hat{C}_{O, 1}^{(1), 0}\left(\hat{\rho}_{1}, t\right)=\left(\frac{a_{1} I_{s s}\left(a_{1} \beta_{1}\right)}{2 \pi \hat{\rho}_{1}}\right) \times \\
\left\{\frac{\alpha_{1} a_{1} I_{s s}\left(a_{1} \beta_{1}\right)}{2 \pi^{\frac{3}{2}} \beta_{1}^{2} \sqrt{D_{O} t}}\left(k_{f, 1}+k_{b, 1}\left(\frac{D_{O}}{D_{R}}\right)^{\frac{3}{2}}\right)\right. \\
\left.-\frac{\alpha_{2} a_{2} I_{s s}\left(a_{2} \beta_{2}\right)}{2 \pi \beta_{1} \beta_{2}}\left(k_{f, 1} \operatorname{erfc}\left(\frac{1}{2 \sqrt{D_{O} t}}\right)+\frac{k_{b, 1} D_{O}}{D_{R}} \operatorname{erfc}\left(\frac{1}{2 \sqrt{D_{R} t}}\right)\right)\right\} .
\end{gathered}
$$


Solution by Laplace transform gives:

$$
\begin{aligned}
& \hat{C}_{O, 1}^{(1)}=\left(\frac{a_{1} I_{s s}\left(a_{1} \beta_{1}\right)}{2 \pi \hat{\rho}_{1}}\right) \times\left\{\frac{\alpha_{1} a_{1} I_{s s}\left(a_{1} \beta_{1}\right)}{2 \pi^{\frac{3}{2}} \beta_{1}^{2} \sqrt{D_{O} t}}\left(k_{f, 1}+k_{b, 1}\left(\frac{D_{O}}{D_{R}}\right)^{\frac{3}{2}}\right) e^{-\frac{\hat{\rho}_{1}^{2}}{4 D_{O} t}}-\right. \\
& \left.\frac{\alpha_{2} a_{2} I_{s s}\left(a_{2} \beta_{2}\right)}{2 \pi \beta_{1} \beta_{2}}\left(k_{f, 1} \operatorname{erfc}\left(\frac{\hat{\rho}_{1}+1}{2 \sqrt{D_{O} t}}\right)+\frac{k_{b, 1} D_{O}}{D_{R}} \operatorname{erfc}\left(\frac{\sqrt{D_{O}}+\sqrt{D_{R}} \hat{\rho}_{1}}{2 \sqrt{D_{O} D_{R} t}}\right)\right)\right\}
\end{aligned}
$$

and, by symmetry, $\hat{C}_{O, 2}^{(1)}$ is given by

$$
\begin{aligned}
& \hat{C}_{O, 2}^{(1)}=\left(\frac{a_{2} I_{s s}\left(a_{2} \beta_{2}\right)}{2 \pi \hat{\rho}_{2}}\right) \times\left\{\frac{\alpha_{2} a_{2} I_{s s}\left(a_{2} \beta_{2}\right)}{2 \pi^{\frac{3}{2}} \beta_{2}^{2} \sqrt{D_{O} t}}\left(k_{f, 2}+k_{b, 2}\left(\frac{D_{O}}{D_{R}}\right)^{\frac{3}{2}}\right) e^{-\frac{\hat{\rho}_{2}^{2}}{4 D_{O} t}}-\right. \\
& \left.\frac{\alpha_{1} a_{1} I_{s s}\left(a_{1} \beta_{1}\right)}{2 \pi \beta_{2} \beta_{1}}\left(k_{f, 2} \operatorname{erfc}\left(\frac{\hat{\rho}_{2}+1}{2 \sqrt{D_{O} t}}\right)+\frac{k_{b, 2} D_{O}}{D_{R}} \operatorname{erfc}\left(\frac{\sqrt{D_{O}}+\sqrt{D_{R}} \hat{\rho}_{2}}{2 \sqrt{D_{O} D_{R} t}}\right)\right)\right\} .
\end{aligned}
$$

Now applying Van Dyke's rule to the reductant, we find that $\hat{C}_{R, 1}^{(1)}\left(\hat{\rho}_{1}, t\right)$ must satisfy:

$$
\begin{aligned}
\frac{D_{R}}{\hat{\rho}_{1}} \frac{\partial^{2}}{\partial \hat{\rho}_{1}^{2}}\left(\hat{\rho}_{1} \hat{C}_{R, 1}^{(1)}\right) & =\frac{\partial \hat{C}_{R, 1}^{(1)}}{\partial t}, & \text { for } \hat{z}>0, \\
\hat{C}_{R, 1}^{(1)} & =0, & \text { at } t=0, \\
\hat{C}_{R, 1}^{(1)} & \sim \hat{C}_{R, 1}^{(1), 0}\left(\hat{\rho}_{1}, t\right), & \text { as } \hat{\rho}_{1} \rightarrow 0, \\
\hat{C}_{R, 1}^{(1)} & \rightarrow 0, & \text { as } \hat{\rho}_{1} \rightarrow \infty,
\end{aligned}
$$

where

$$
\begin{gathered}
\hat{C}_{R, 1}^{(1), 0}\left(\hat{\rho}_{1}, t\right)=-\left(\frac{a_{1} I_{s s}\left(a_{1} \beta_{1}\right)}{2 \pi \hat{\rho}_{1}}\right) \times \\
\frac{D_{O}}{D_{R}}\left\{\frac{\alpha_{1} a_{1} I_{s s}\left(a_{1} \beta_{1}\right)}{2 \pi^{\frac{3}{2}} \beta_{1}^{2} \sqrt{D_{O} t}}\left(k_{f, 1}+k_{b, 1}\left(\frac{D_{O}}{D_{R}}\right)^{\frac{3}{2}}\right)-\right. \\
\left.\frac{\alpha_{2} a_{2} I_{s s}\left(a_{2} \beta_{2}\right)}{2 \pi \beta_{1} \beta_{2}}\left(k_{f, 1} \operatorname{erfc}\left(\frac{1}{2 \sqrt{D_{O} t}}\right)+\frac{k_{b, 1} D_{O}}{D_{R}} \operatorname{erfc}\left(\frac{1}{2 \sqrt{D_{R} t}}\right)\right)\right\} .
\end{gathered}
$$


Using the Laplace transform, this problem has the solution

$$
\begin{aligned}
& \hat{C}_{R, 1}^{(1)}=-\left(\frac{a_{1} I_{s s}\left(a_{1} \beta_{1}\right)}{2 \pi \hat{\rho}_{1}}\right) \frac{D_{O}}{D_{R}} \times \\
& \left\{\frac{\alpha_{1} a_{1} I_{s s}\left(a_{1} \beta_{1}\right)}{2 \pi^{\frac{3}{2}} \beta_{1}^{2} \sqrt{D_{O} t}}\left(k_{f, 1}+k_{b, 1}\left(\frac{D_{O}}{D_{R}}\right)^{\frac{3}{2}}\right) e^{-\frac{\hat{\rho}_{1}^{2}}{4 D_{R} t}}-\right. \\
& \left.\frac{\alpha_{2} a_{2} I_{s s}\left(a_{2} \beta_{2}\right)}{2 \pi \beta_{1} \beta_{2}}\left(k_{f, 1} \operatorname{erfc}\left(\frac{\sqrt{D_{O}} \hat{\rho}_{1}+\sqrt{D_{R}}}{2 \sqrt{D_{O} D_{R} t}}\right)+\frac{k_{b, 1} D_{O}}{D_{R}} \operatorname{erfc}\left(\frac{\hat{\rho}_{1}+1}{2 \sqrt{D_{R} t}}\right)\right)\right\},
\end{aligned}
$$

and, by symmetry,

$$
\begin{gathered}
\hat{C}_{R, 2}^{(1)}=-\left(\frac{a_{2} I_{s s}\left(a_{2} \beta_{2}\right)}{2 \pi \hat{\rho}_{2}}\right) \frac{D_{O}}{D_{R}} \times \\
\left\{\frac{\alpha_{2} a_{2} I_{s s}\left(a_{2} \beta_{2}\right)}{2 \pi^{\frac{3}{2}} \beta_{2}^{2} \sqrt{D_{O} t}}\left(k_{f, 2}+k_{b, 2}\left(\frac{D_{O}}{D_{R}}\right)^{\frac{3}{2}}\right) e^{-\frac{\hat{\rho}_{2}^{2}}{4 D_{R} t}}-\right. \\
\left.\frac{\alpha_{1} a_{1} I_{s s}\left(a_{1} \beta_{1}\right)}{2 \pi \beta_{1} \beta_{2}}\left(k_{f, 2} \operatorname{erfc}\left(\frac{\sqrt{D_{O}} \hat{\rho}_{2}+\sqrt{D_{R}}}{2 \sqrt{D_{O} D_{R} t}}\right)+\frac{k_{b, 2} D_{O}}{D_{R}} \operatorname{erfc}\left(\frac{\hat{\rho}_{2}+1}{2 \sqrt{D_{R} t}}\right)\right)\right\} .
\end{gathered}
$$

\subsubsection{Second-order inner solution}

To find the second-order inner solution, let us find the three-term inner expansion of the two-term outer expansion near disk 1 and apply Van Dyke's rule. To recap, the two-term outer expansions for the oxidant and reductant are given by

$$
\begin{aligned}
& \hat{C}_{O} \sim \epsilon\left(\hat{C}_{O, 1}^{(0)}\left(\hat{\rho}_{1}, t\right)+\hat{C}_{O, 2}^{(0)}\left(\hat{\rho}_{2}, t\right)\right)+\epsilon^{2}\left(\hat{C}_{O, 1}^{(1)}\left(\hat{\rho}_{1}, t\right)+\hat{C}_{O, 2}^{(1)}\left(\hat{\rho}_{2}, t\right)\right), \\
& \hat{C}_{R} \sim \epsilon\left(\hat{C}_{R, 1}^{(0)}\left(\hat{\rho}_{1}, t\right)+\hat{C}_{R, 2}^{(0)}\left(\hat{\rho}_{2}, t\right)\right)+\epsilon^{2}\left(\hat{C}_{R, 1}^{(1)}\left(\hat{\rho}_{1}, t\right)+\hat{C}_{R, 2}^{(1)}\left(\hat{\rho}_{2}, t\right)\right) .
\end{aligned}
$$

Here $\hat{C}_{O, j}^{(0)}$ and $\hat{C}_{R, j}^{(0)}$ are given by (29a) and (29b) respectively; $\hat{C}_{O, 1}^{(1)}$ and $\hat{C}_{O, 2}^{(1)}$ are given by (39a) and (39b); and, $\hat{C}_{R, 1}^{(1)}$ and $\hat{C}_{R, 2}^{(1)}$ are given by (45a) and (45b).

Finding the three-term inner expansions of (46a) and (46b), and using the $O\left(\epsilon^{2}\right)$ terms to obtain our matching conditions, we find that $C_{O, 1}^{(2)}$ and $C_{R, 1}^{(2)}$ satisfy the following problem:

$$
D_{O} \nabla^{2} C_{O, 1}^{(2)}=\frac{\partial C_{O, 1}^{(0)}}{\partial t}=0, \quad D_{R} \nabla^{2} C_{R, 1}^{(2)}=\frac{\partial C_{R, 1}^{(0)}}{\partial t}=0
$$

with boundary conditions:

$$
\frac{\partial C_{O, 1}^{(2)}}{\partial z}=\frac{\partial C_{R, 1}^{(2)}}{\partial z}=0, \quad \text { for } z=0, r_{1}>a_{1}
$$




$$
\left.\left.\begin{array}{c}
-\frac{\partial C_{O, 1}^{(2)}}{\partial z}=-k_{f, 1} C_{O, 1}^{(2)}+k_{b, 1} C_{R, 1}^{(2)}, \\
-\frac{\partial C_{O, 1}^{(2)}}{\partial z}=\frac{D_{R}}{D_{O}} \frac{\partial C_{R, 1}^{(2)}}{\partial z},
\end{array}\right\} \quad \text { for } z=0, r_{1} \leq a_{1}, \quad \text { (47 }\right)
$$

The functions $C_{O, 1}^{(2), \infty}\left(r_{1}, \theta_{1}, t\right)$ and $C_{R, 1}^{(2), \infty}\left(r_{1}, \theta_{1}, t\right)$ are determined from the matching condition to be:

$$
\begin{aligned}
& C_{O, 1}^{(2), \infty}\left(r_{1}, \theta_{1}, t\right)=G_{O, 1}^{\infty}(t)-F_{O, 1}^{\infty}(t) r_{1} \cos \theta_{1}, \\
& C_{R, 1}^{(2), \infty}\left(r_{1}, \theta_{1}, t\right)=G_{R, 1}^{\infty}(t)-F_{R, 1}^{\infty}(t) r_{1} \cos \theta_{1},
\end{aligned}
$$

where

$$
\begin{aligned}
& F_{O, 1}^{\infty}(t)=\frac{\alpha_{2} a_{2} I_{s s}\left(a_{2} \beta_{2}\right)}{2 \pi \beta_{2}}\left[\operatorname{erfc}\left(\frac{1}{2 \sqrt{D_{O} t}}\right)+\frac{e^{-\frac{1}{4 D_{O} t}}}{\sqrt{\pi D_{O} t}}\right], \\
& F_{R, 1}^{\infty}(t)=\frac{\alpha_{2} a_{2} I_{s s}\left(a_{2} \beta_{2}\right)}{2 \pi \beta_{2}}\left[\operatorname{erfc}\left(\frac{1}{2 \sqrt{D_{R} t}}\right)+\frac{e^{-\frac{1}{4 D_{R} t}}}{\sqrt{\pi D_{R} t}}\right],
\end{aligned}
$$

and

$$
\begin{gathered}
G_{O, 1}^{\infty}(t)=\frac{a_{1} a_{2} \alpha_{2} I_{s s}\left(a_{1} \beta_{1}\right) I_{s s}\left(a_{2} \beta_{2}\right)}{4 \pi^{2} \beta_{1} \beta_{2}}\left[k_{f, 1} \frac{e^{-\frac{1}{4 D_{O} t}}}{\sqrt{\pi D_{O} t}}+\frac{k_{b, 1} D_{O}}{D_{R}} \frac{e^{-\frac{1}{4 D_{R} t}}}{\sqrt{\pi D_{O} t}}\right] \\
-\frac{a_{1} a_{2} \alpha_{1} I_{s s}\left(a_{1} \beta_{1}\right) I_{s s}\left(a_{2} \beta_{2}\right)}{4 \pi^{2} \beta_{1} \beta_{2}}\left[k_{f, 2} \operatorname{erfc}\left(\frac{1}{\sqrt{D_{O} t}}\right)+\frac{k_{b, 2} D_{O}}{D_{R}} \operatorname{erfc}\left(\frac{\sqrt{D_{O}}+\sqrt{D_{R}}}{2 \sqrt{D_{R} D_{O} t}}\right)\right] \\
+\frac{a_{2}^{2} \alpha_{2} I_{s s}\left(a_{2} \beta_{2}\right)^{2}}{4 \pi^{2} \beta_{2}^{2}}\left(k_{f, 2}+k_{b, 2}\left(\frac{D_{O}}{D_{R}}\right)^{\frac{3}{2}}\right) \frac{e^{-\frac{1}{4 D^{t} t}}}{\sqrt{\pi D_{O} t}}, \quad \text { (51a) }
\end{gathered}
$$

and

$$
\begin{gathered}
G_{R, 1}^{\infty}(t)=\frac{a_{1} a_{2} \alpha_{2} I_{s s}\left(a_{1} \beta_{1}\right) I_{s s}\left(a_{2} \beta_{2}\right)}{4 \pi^{2} \beta_{1} \beta_{2}}\left[k_{f, 1} \frac{e^{-\frac{1}{4 D_{O} t}}}{\sqrt{\pi D_{R} t}}+\frac{k_{b, 1} D_{O}}{D_{R}} \frac{e^{-\frac{1}{4 D_{R} t}}}{\sqrt{\pi D_{R} t}}\right] \\
-\frac{a_{1} a_{2} \alpha_{1} I_{s s}\left(a_{1} \beta_{1}\right) I_{s s}\left(a_{2} \beta_{2}\right)}{4 \pi^{2} \beta_{1} \beta_{2}}\left[k_{f, 2} \operatorname{erfc}\left(\frac{\sqrt{D_{O}}+\sqrt{D_{R}}}{2 \sqrt{D_{R} D_{O} t}}\right)+\frac{k_{b, 2} D_{O}}{D_{R}} \operatorname{erfc}\left(\frac{1}{\sqrt{D_{R} t}}\right)\right] \\
+\frac{a_{2}^{2} \alpha_{2} I_{s s}\left(a_{2} \beta_{2}\right)^{2}}{4 \pi^{2} \beta_{2}^{2}}\left(k_{f, 2}+k_{b, 2}\left(\frac{D_{O}}{D_{R}}\right)^{\frac{3}{2}}\right) \frac{e^{-\frac{1}{4 D_{R} t}}}{\sqrt{\pi D_{O} t}} .
\end{gathered}
$$

Suppose that we let:

$$
\begin{aligned}
C_{O, 1}^{(2)} & =G_{O, 1}\left(r_{1}, z, t\right)-F_{O, 1}\left(r_{1}, z, t\right) \cos \theta_{1}, \\
C_{R, 1}^{(2)} & =G_{R, 1}\left(r_{1}, z, t\right)-F_{R, 1}\left(r_{1}, z, t\right) \cos \theta_{1} .
\end{aligned}
$$


Then, the solutions for $F_{O, 1}$ and $F_{R, 1}$ are unnecessary for the calculation of the current, as they do not contribute to the current through the disk, since $\cos \theta_{1}$ integrates to zero. Therefore it remains to find the solutions for $G_{O, 1}$ and $G_{R, 1}$, which solve

$$
\nabla^{2} G_{O, 1}=0, \quad \nabla^{2} G_{R, 1}=0,
$$

with boundary conditions:

$$
\left.\begin{array}{c}
\frac{\partial G_{O, 1}}{\partial z}=\frac{\partial G_{R, 1}}{\partial z}=0, \quad \text { for } z=0, r_{1}>a_{1}, \\
-\frac{\partial G_{O, 1}}{\partial z}=-k_{f, 1} G_{O, 1}+k_{b, 1} G_{R, 1}, \\
-\frac{\partial G_{O, 1}}{\partial z}=\frac{D_{R}}{D_{O}} \frac{\partial G_{R, 1}}{\partial z},
\end{array}\right\} \text { for } z=0, r_{1} \leq a_{1},
$$

Again, the following expression must be satisfied:

$$
G_{O, 1}+\frac{D_{R}}{D_{O}} G_{R, 1}=G_{O, 1}^{\infty}(t)-G_{R, 1}^{\infty}(t),
$$

and the solutions for $G_{O, 1}$ and $G_{R, 1}$ are given by

$$
\begin{aligned}
G_{O, 1}\left(r_{1}, z, t\right)= & G_{O, 1}^{\infty}(t)- \\
& \left(\frac{k_{f, 1} G_{O, 1}^{\infty}(t)+\frac{k_{b, 1} D_{O}}{D_{R}} G_{R, 1}^{\infty}(t)}{\beta_{1}}\right) \chi\left(a_{1} \beta_{1} ; \frac{r_{1}}{a_{1}}, \frac{z}{a_{1}}\right), \\
G_{R, 1}\left(r_{1}, z, t\right)= & -\frac{D_{O}}{D_{R}} G_{R, 1}^{\infty}(t)+ \\
& \frac{D_{O}}{D_{R}}\left(\frac{k_{f, 1} G_{O, 1}^{\infty}(t)+\frac{k_{b, 1} D_{O}}{D_{R}} G_{R, 1}^{\infty}(t)}{\beta_{1}}\right) \chi\left(a_{1} \beta_{1} ; \frac{r_{1}}{a_{1}}, \frac{z}{a_{1}}\right) .
\end{aligned}
$$

The equivalent solutions for $G_{O, 2}$ and $G_{R, 2}$ in the vicinity of disk 2 can be obtained by interchanging the subscripts 1 and 2 .

\subsection{Analytical expression for the Faradaic current through each disk}

The Faradaic current through disk 1 is given by expression (10) with $j=1$, so that:

$$
\begin{aligned}
I_{1}(t) & =\int_{0}^{2 \pi} \int_{0}^{a_{1}} \frac{\partial C_{O, 1}}{\partial z}\left(r_{1}, 0, \theta_{1}, t\right) r_{1} d r_{1} d \theta_{1}, \\
& =\left.\int_{0}^{2 \pi} \int_{0}^{a_{1}}\left(\frac{\partial C_{O, 1}^{(0)}}{\partial z}+\epsilon \frac{\partial C_{O, 1}^{(1)}}{\partial z}+\epsilon^{2} \frac{\partial C_{O, 1}^{(2)}}{\partial z}\right)\right|_{z=0} r_{1} d r_{1} d \theta_{1}+O\left(\epsilon^{3}\right) .
\end{aligned}
$$


We substitute in the expressions for $C_{O, 1}^{(0)}, C_{O, 1}^{(1)}$ and $C_{O, 1}^{(2)}$ from (18a) (with $j=1$ ), (35a) and (52a) respectively, and use the fact that (cf. equation (23)):

$$
\left.\int_{0}^{2 \pi} \int_{0}^{a_{1}} \frac{\partial \chi\left(a_{1} \beta_{1} ; \frac{r_{1}}{a_{1}}, \frac{z}{a_{1}}\right)}{\partial z}\right|_{z=0} r_{1} d r_{1} d \theta_{1}=a_{1} I_{s s}\left(a_{1} \beta_{1}\right) .
$$

These steps give:

$$
\begin{aligned}
I_{1}(t)=\frac{a_{1} I_{s s}\left(a_{1} \beta_{1}\right)}{\beta_{1}}\{ & \alpha_{1}-\epsilon\left(k_{f, 1} C_{O, 1}^{(1), \infty}(t)+\frac{k_{b, 1} D_{O}}{D_{R}} C_{R, 1}^{(1), \infty}(t)\right) \\
& \left.-\epsilon^{2}\left(k_{f, 1} G_{O, 1}^{\infty}(t)+\frac{k_{b, 1} D_{O}}{D_{R}} G_{R, 1}^{\infty}(t)\right)\right\}+O\left(\epsilon^{3}\right),
\end{aligned}
$$

where $C_{O, 1}^{(1), \infty}(t), C_{R, 1}^{(1), \infty}(t), G_{O, 1}^{\infty}(t)$ and $G_{R, 1}^{\infty}(t)$ are given by (32a), (32b), (51a) and $(51 \mathrm{~b})$ respectively.

Converting back into dimensional variables gives the main analytical result of this article. The dimensional Faradaic current through disk 1 is given by:

$$
\begin{aligned}
\tilde{I}_{1}(\tilde{t}) \approx\left(\frac{n \tilde{F} \tilde{D}_{O} \tilde{a}_{1} I_{s s}\left(a_{1} \beta_{1}\right)}{\tilde{\mathcal{K}}_{1}}\right)\left\{\tilde{C}_{O}^{*}-\frac{\tilde{k}_{b, 1}}{\tilde{k}_{f, 1}} \tilde{C}_{R}^{*}\right. \\
-\left(\tilde{C}_{O, 1}^{(1), \infty}(\tilde{t})+\frac{\tilde{k}_{b, 1} \tilde{D}_{O}}{\tilde{k}_{f, 1} \tilde{D}_{R}} \tilde{C}_{R, 1}^{(1), \infty}(\tilde{t})\right) \\
\\
\left.-\left(\tilde{G}_{O, 1}^{\infty}(\tilde{t})+\frac{\tilde{k}_{b, 1} \tilde{D}_{O}}{\tilde{k}_{f, 1} \tilde{D}_{R}} \tilde{G}_{R, 1}^{\infty}(\tilde{t})\right)\right\},
\end{aligned}
$$

where

$$
\begin{aligned}
& \tilde{C}_{O, 1}^{(1), \infty}(\tilde{t})=\frac{\tilde{a}_{1} \tilde{\mathcal{C}}_{1} I_{s s}\left(a_{1} \beta_{1}\right)}{2 \pi^{\frac{3}{2}} \tilde{\mathcal{K}}_{1} \sqrt{\tilde{D}_{O} \tilde{t}}}-\frac{\tilde{a}_{2} \tilde{\mathcal{C}}_{2} I_{s s}\left(a_{2} \beta_{2}\right)}{2 \pi \tilde{\mathcal{K}}_{2} \tilde{L}} \operatorname{erfc}\left(\frac{\tilde{L}}{2 \sqrt{\tilde{D}_{O} \tilde{t}}}\right), \\
& \tilde{C}_{R, 1}^{(1), \infty}(\tilde{t})=\frac{\tilde{a}_{1} \tilde{\mathcal{C}}_{1} I_{s s}\left(a_{1} \beta_{1}\right)}{2 \pi^{\frac{3}{2}} \tilde{\mathcal{K}}_{1} \sqrt{\tilde{D}_{R} \tilde{t}}}-\frac{\tilde{a}_{2} \tilde{\mathcal{C}}_{2} I_{s s}\left(a_{2} \beta_{2}\right)}{2 \pi \tilde{\mathcal{K}}_{2} \tilde{L}} \operatorname{erfc}\left(\frac{\tilde{L}}{2 \sqrt{\tilde{D}_{R} \tilde{t}}}\right),
\end{aligned}
$$


and

$$
\begin{aligned}
& \tilde{G}_{O, 1}^{\infty}(\tilde{t})=\frac{\tilde{a}_{2}^{2} \tilde{\mathcal{C}}_{2} I_{s s}\left(a_{2} \beta_{2}\right)^{2}}{4 \pi^{\frac{5}{2}} \tilde{\mathcal{K}}_{2}^{2} \tilde{L} \sqrt{\tilde{D}_{O} \tilde{t}}}\left(1+\frac{\tilde{k}_{b, 2}}{\tilde{k}_{f, 2}}\left(\frac{\tilde{D}_{O}}{\tilde{D}_{R}}\right)^{\frac{3}{2}}\right) e^{-\frac{\tilde{L}^{2}}{4 \tilde{D}_{O} t}} \\
& +\frac{\tilde{a}_{2} \tilde{a}_{1} \tilde{\mathcal{C}}_{2} I_{s s}\left(a_{1} \beta_{1}\right) I_{s s}\left(a_{2} \beta_{2}\right)}{4 \pi^{\frac{5}{2}} \tilde{\mathcal{K}}_{1} \tilde{\mathcal{K}}_{2} \tilde{L} \sqrt{\tilde{D}_{O} \tilde{t}}}\left(e^{-\frac{\tilde{L}^{2}}{4 \tilde{D}_{O} \tilde{t}}}+\frac{\tilde{k}_{b, 1} \tilde{D}_{O}}{\tilde{k}_{f, 1} \tilde{D}_{R}} e^{-\frac{\tilde{L}^{2}}{4 \tilde{D}_{R} t}}\right) \\
& -\frac{\tilde{a}_{1} \tilde{a}_{2} \tilde{\mathcal{C}}_{1} I_{s s}\left(a_{2} \beta_{2}\right) I_{s s}\left(a_{1} \beta_{1}\right)}{4 \pi^{2} \tilde{\mathcal{K}}_{2} \tilde{\mathcal{K}}_{1} \tilde{L}^{2}}\left\{\operatorname{erfc}\left(\frac{\tilde{L}}{\sqrt{\tilde{D}_{O} \tilde{t}}}\right)\right. \\
& \left.+\frac{\tilde{k}_{b, 2} \tilde{D}_{O}}{\tilde{k}_{f, 2} \tilde{D}_{R}} \operatorname{erfc}\left(\frac{\tilde{L} \sqrt{\tilde{D}_{O}}+\tilde{L} \sqrt{\tilde{D}_{R}}}{2 \sqrt{\tilde{D}_{O} \tilde{D}_{R} \tilde{t}}}\right)\right\} \\
& \tilde{G}_{R, 1}^{\infty}(\tilde{t})=\frac{\tilde{a}_{2}^{2} \tilde{\mathcal{C}}_{2} I_{s s}\left(a_{2} \beta_{2}\right)^{2}}{4 \pi^{\frac{5}{2}} \tilde{\mathcal{K}}_{2}^{2} \tilde{L} \sqrt{\tilde{D}_{O} \tilde{t}}}\left(1+\frac{\tilde{k}_{b, 2}}{\tilde{k}_{f, 2}}\left(\frac{\tilde{D}_{O}}{\tilde{D}_{R}}\right)^{\frac{3}{2}}\right) e^{-\frac{\tilde{L}^{2}}{4 \tilde{D}_{R} t}} \\
& +\frac{\tilde{a}_{2} \tilde{a}_{1} \tilde{\mathcal{C}}_{2} I_{s s}\left(a_{1} \beta_{1}\right) I_{s s}\left(a_{2} \beta_{2}\right)}{4 \pi^{\frac{5}{2}} \tilde{\mathcal{K}}_{1} \tilde{\mathcal{K}}_{2} \tilde{L} \sqrt{\tilde{D}_{R} \tilde{t}}}\left(e^{-\frac{\tilde{L}^{2}}{4 \tilde{D}_{O} t}}+\frac{\tilde{k}_{b, 1} \tilde{D}_{O}}{\tilde{k}_{f, 1} \tilde{D}_{R}} e^{-\frac{\tilde{L}^{2}}{4 \tilde{D}_{R} t}}\right) \\
& -\frac{\tilde{a}_{1} \tilde{a}_{2} \tilde{\mathcal{C}}_{1} I_{s s}\left(a_{2} \beta_{2}\right) I_{s s}\left(a_{1} \beta_{1}\right)}{4 \pi^{2} \tilde{\mathcal{K}}_{2} \tilde{\mathcal{K}}_{1} \tilde{L}^{2}}\left\{\operatorname{erfc}\left(\frac{\sqrt{\tilde{D}_{O}} \tilde{L}+\sqrt{\tilde{D}_{R}} \tilde{L}}{2 \sqrt{\tilde{D}_{O} \tilde{D}_{R} \tilde{t}}}\right)\right. \\
& \left.+\frac{\tilde{k}_{b, 2} \tilde{D}_{O}}{\tilde{k}_{f, 2} \tilde{D}_{R}} \operatorname{erfc}\left(\frac{\tilde{L}}{\sqrt{\tilde{D}_{R} \tilde{t}}}\right)\right\} \text {, }
\end{aligned}
$$

and

$$
\begin{gathered}
\tilde{\mathcal{C}}_{j}=\tilde{C}_{O}^{*}-\frac{\tilde{k}_{b, j}}{\tilde{k}_{f, j}} \tilde{C}_{R}^{*}, \\
\tilde{\mathcal{K}}_{j}=1+\frac{\tilde{k}_{b, j} \tilde{D}_{O}}{\tilde{k}_{f, j} \tilde{D}_{R}}, \\
a_{j} \beta_{j}=\frac{\tilde{k}_{f, j} \tilde{a}_{j}}{\tilde{D}_{O}}+\frac{\tilde{k}_{b, j} \tilde{a}_{j}}{\tilde{D}_{R}} .
\end{gathered}
$$

The equivalent expression for the current through disk 2 can be obtained by interchanging the subscripts 1 and 2 in (60). We recall that the factor $I_{s s}(\beta)$ in the above expressions can be derived for $0 \leq \beta \leq 500$ from the working curve in the Supplementary Data to our previous paper [18], and for $\beta>500$ from the asymptotic expression (24) derived by Phillips [23]. We also emphasize that the percentage error in expression (60a) for the current is $O\left(\epsilon^{3}\right)$, i.e. $O\left((\tilde{a} / \tilde{L})^{3}\right)$, where $\tilde{a}$ is the typical radius of the electrodes and $\tilde{L}$ is the distance between their centres. For (60a) to be accurate, this error must be small. In addition, it is only valid for time-scales such that the diffusion layers of the electrodes have started to interact, i.e. for $\tilde{t}$ such that condition (2) holds. 


\section{Results and discussion}

In this section, we verify that the solution for the dimensional current (60) encompasses the solution derived in our previous paper [18] for a single disk, and we discuss the quasi-reversible steady state, reversible reactions and diffusion-limited reactions. In the latter case, we show that our expression reduces to Strieder's solution in [15] for the steady-state current response of two disks with identical radii. We also highlight the particular case of a generator-collector system, for which (60) reduces to simple expressions for the current through each electrode and the steady-state collection efficiency. Finally, we illustrate the effects of shielding and feedback on the current responses, and show that the impact of unequal diffusion coefficients of the oxidant and reductant can be substantial.

\subsection{Single disk response}

In the limit that $\tilde{a}_{2} \rightarrow 0$ in (60), the second disk vanishes and we recover the time-dependent response of the single disk, namely:

$$
\begin{aligned}
& \tilde{I}_{1}(\tilde{t}) \approx n \tilde{F} \tilde{a}_{1} \tilde{D}_{O} \tilde{D}_{R} I_{s s}\left(a_{1} \beta_{1}\right)\left(\frac{\tilde{k}_{f, 1} \tilde{C}_{O}^{*}-\tilde{k}_{b, 1} \tilde{C}_{R}^{*}}{\tilde{k}_{f, 1} \tilde{D}_{R}+\tilde{k}_{b, 1} \tilde{D}_{O}}\right) \times \\
& {\left[1-\frac{\tilde{a}_{1} I_{s s}\left(a_{1} \beta_{1}\right)}{2 \pi^{\frac{3}{2}} \sqrt{\tilde{D}_{O} \tilde{D}_{R} \tilde{t}}}\left(\frac{\tilde{k}_{f, 1} \tilde{D}_{R}^{\frac{3}{2}}+\tilde{k}_{b, 1} \tilde{D}_{O}^{\frac{3}{2}}}{\tilde{k}_{f, 1} \tilde{D}_{R}+\tilde{k}_{b, 1} \tilde{D}_{O}}\right)\right], }
\end{aligned}
$$

which agrees with the single-disk response derived in our previous article [18].

\subsection{Quasi-reversible steady-state response}

Letting $\tilde{t} \rightarrow \infty$ in (60) gives the steady-state response, which is equal to:

$$
\begin{aligned}
\tilde{I}_{1}(\infty) \approx\left(\frac{n \tilde{F} \tilde{D}_{O} \tilde{a}_{1} I_{s s}\left(a_{1} \beta_{1}\right)}{\tilde{\mathcal{K}}_{1}}\right)\left\{\tilde{C}_{O}^{*}-\frac{\tilde{k}_{b, 1}}{\tilde{k}_{f, 1}} \tilde{C}_{R}^{*}\right. \\
\left.\quad+\frac{\tilde{a}_{2} \tilde{\mathcal{C}}_{2} \tilde{\mathcal{K}}_{1} I_{s s}\left(a_{2} \beta_{2}\right)}{2 \pi \tilde{\mathcal{K}}_{2} \tilde{L}}+\frac{\tilde{a}_{1} \tilde{a}_{2} \tilde{\mathcal{C}}_{1} I_{s s}\left(a_{2} \beta_{2}\right) I_{s s}\left(a_{1} \beta_{1}\right)}{4 \pi^{2} \tilde{L}^{2}}\right\} .
\end{aligned}
$$

\subsection{Reversible reactions}

If the redox reactions at both the electrodes are reversible, then this corresponds to letting $\tilde{k}_{f, j}, \tilde{k}_{b, j} \rightarrow \infty$ such that $\tilde{k}_{b, j} / \tilde{k}_{f, j}=O(1)$. Since $I_{s s}\left(a_{j} \beta_{j}\right) \rightarrow-4$ as $\beta_{j} \rightarrow \infty$ (cf. (24)), and expression (60) is already written in terms of the ratio $\tilde{k}_{b, j} / \tilde{k}_{f, j}$, the analytical expression for a reversible current is easily obtained by setting $I_{s s}\left(a_{j} \beta_{j}\right)=-4$ in (60). The reversible steady-state current has the simple analytical 
form:

$$
\begin{aligned}
\tilde{I}_{1}(\infty) \approx-\left(\frac{4 n \tilde{F} \tilde{D}_{O} \tilde{D}_{R} \tilde{a}_{1}}{\tilde{D}_{R}+\frac{\tilde{k}_{b, 1}}{\tilde{k}_{f, 1}} \tilde{D}_{O}}\right)\left\{\tilde{C}_{O}^{*}-\frac{\tilde{k}_{b, 1}}{\tilde{k}_{f, 1}} \tilde{C}_{R}^{*}\right. & \\
-\frac{2 \tilde{a}_{2}\left(\tilde{C}_{O}^{*}-\frac{\tilde{k}_{b, 2}}{\tilde{k}_{f, 2}} \tilde{C}_{R}^{*}\right)\left(1+\frac{\tilde{k}_{b, 1} \tilde{D}_{O}}{\tilde{k}_{f, 1} \tilde{D}_{R}}\right)}{\pi\left(1+\frac{\tilde{k}_{b, 2} \tilde{D}_{O}}{k_{f, 2} \tilde{D}_{R}}\right) \tilde{L}} & \\
& \left.+\frac{4 \tilde{a}_{1} \tilde{a}_{2}\left(\tilde{C}_{O}^{*}-\frac{\tilde{k}_{b, 1} \tilde{C}_{R, 1}^{*}}{\tilde{C}_{f,}}\right)}{\pi^{2} \tilde{L}^{2}}\right\} .
\end{aligned}
$$

\subsection{Diffusion-limited reactions due to extreme polarisation}

For a diffusion-limited reduction reaction, the potential at both the electrodes is set to a level such that $\tilde{k}_{f, j} \rightarrow \infty$ and $\tilde{k}_{b, j} \rightarrow 0$. From (24), this means that $I_{s s}\left(a_{j} \beta_{j}\right)=-4$, and (60) simplifies to:

$$
\begin{aligned}
\tilde{I}_{1}(\tilde{t}) \approx-4 n \tilde{F} \tilde{D}_{O} \tilde{a}_{1} \tilde{C}_{O}^{*}\left\{1+\frac{2 \tilde{a}_{1}}{\pi^{\frac{3}{2}} \sqrt{\tilde{D}_{O} \tilde{t}}}-\frac{2 \tilde{a}_{2}}{\pi \tilde{L}} \operatorname{erfc}\left(\frac{\tilde{L}}{2 \sqrt{\tilde{D}_{O} \tilde{t}}}\right)\right. \\
\left.-\frac{4 \tilde{a}_{2}\left(\tilde{a}_{2}+\tilde{a}_{1}\right)}{\pi^{\frac{5}{2}} \tilde{L} \sqrt{\tilde{D}_{O} \tilde{t}}} e^{-\frac{\tilde{L}^{2}}{4 \bar{D}}{ }^{\tilde{t}}}+\frac{4 \tilde{a}_{1} \tilde{a}_{2}}{\pi^{2} \tilde{L}^{2}} \operatorname{erfc}\left(\frac{\tilde{L}}{\sqrt{\tilde{D}_{O} \tilde{t}}}\right)\right\},
\end{aligned}
$$

with corresponding steady state:

$$
\tilde{I}_{1}(\infty) \approx-4 n \tilde{F} \tilde{D}_{O} \tilde{a}_{1} \tilde{C}_{O}^{*}\left\{1-\frac{2 \tilde{a}_{2}}{\pi \tilde{L}}+\frac{4 \tilde{a}_{1} \tilde{a}_{2}}{\pi^{2} \tilde{L}^{2}}\right\}
$$

For two electrodes with identical radii $\tilde{a}$, this steady state simplifies to

$$
\tilde{I}_{1}(\infty) \approx-4 n \tilde{F} \tilde{D}_{O} \tilde{a} \tilde{C}_{O}^{*}\left\{1-\frac{2 \tilde{a}}{\pi \tilde{L}}+\frac{4 \tilde{a}^{2}}{\pi^{2} \tilde{L}^{2}}\right\},
$$

which agrees with the first three terms in the solution derived by Strieder [15].

Similarly, for a diffusion-limited oxidation reaction, the potential at both the electrodes is set to a level such that $\tilde{k}_{f, j} \rightarrow 0$ and $\tilde{k}_{b, j} \rightarrow \infty$. This again means that $I_{s s}\left(a_{j} \beta_{j}\right)=-4$ and (60) simplifies to:

$$
\begin{aligned}
\tilde{I}_{1}(\tilde{t}) \approx 4 n \tilde{F} \tilde{D}_{R} \tilde{a}_{1} \tilde{C}_{R}^{*}\left\{1+\frac{2 \tilde{a}_{1}}{\pi^{\frac{3}{2}} \sqrt{\tilde{D}_{R} \tilde{t}}}-\frac{2 \tilde{a}_{2}}{\pi \tilde{L}} \operatorname{erfc}\left(\frac{\tilde{L}}{2 \sqrt{\tilde{D}_{R} \tilde{t}}}\right)\right. \\
\left.-\frac{4 \tilde{a}_{2}\left(\tilde{a}_{2}+\tilde{a}_{1}\right)}{\pi^{\frac{5}{2}} \tilde{L} \sqrt{\tilde{D}_{R} \tilde{t}}} e^{-\frac{\tilde{L}^{2}}{4 \bar{D}_{R} t}}+\frac{4 \tilde{a}_{1} \tilde{a}_{2}}{\pi^{2} \tilde{L}^{2}} \operatorname{erfc}\left(\frac{\tilde{L}}{\sqrt{\tilde{D}_{R} \tilde{t}}}\right)\right\}
\end{aligned}
$$


with steady state:

$$
\tilde{I}_{1}(\infty) \approx 4 n \tilde{F} \tilde{D}_{R} \tilde{a}_{1} \tilde{C}_{R}^{*}\left\{1-\frac{2 \tilde{a}_{2}}{\pi \tilde{L}}+\frac{4 \tilde{a}_{1} \tilde{a}_{2}}{\pi^{2} \tilde{L}^{2}}\right\}
$$

As expected, (67) and (68) are equivalent to (64) and (65) with the sign changed and $\tilde{C}_{O}^{*}, \tilde{D}_{O}$ replaced by $\tilde{C}_{R}^{*}, \tilde{D}_{R}$. Also, note that the shielding effects are very apparent in the expressions for the steady-state currents (65) and (68), corresponding to the negative second term in the curly brackets.

\subsection{Generator-collector systems}

A system of two disk electrodes can be set up as a generator-collector system. For a reduction reaction, we assume that the bulk concentration of the reductant is zero, $\tilde{C}_{R}^{*}=0$, so that the bulk solution only contains oxidant, $\tilde{C}_{O}^{*}$. The oxidant is reduced at the generator disk 1 , which is set to an extreme polarisation such that the current is diffusion-limited i.e. $\tilde{k}_{f, 1} \rightarrow \infty$ and $\tilde{k}_{b, 1} \rightarrow 0$. This produces the reductant which diffuses to the collector disk 2 to be oxidised, since the collector is set at the opposite diffusion-limited extreme polarisation, i.e. $\tilde{k}_{f, 2} \rightarrow 0$ and $\tilde{k}_{b, 2} \rightarrow \infty$. Then the current through the generator disk 1 simplifies to

$$
\tilde{I}_{1}(\tilde{t}) \approx-4 n \tilde{F} \tilde{D}_{O} \tilde{a}_{1} \tilde{C}_{O}^{*}\left\{1+\frac{2 \tilde{a}_{1}}{\pi^{\frac{3}{2}} \sqrt{\tilde{D}_{O} \tilde{t}}}+\frac{4 \tilde{a}_{1} \tilde{a}_{2}}{\pi^{2} \tilde{L}^{2}} \operatorname{erfc}\left(\frac{\tilde{L} \sqrt{\tilde{D}_{O}}+\tilde{L} \sqrt{\tilde{D}_{R}}}{2 \sqrt{\tilde{D}_{O} \tilde{D}_{R} \tilde{t}}}\right)\right\}
$$

and that through the collector disk 2:

$$
\tilde{I}_{2}(\tilde{t}) \approx \frac{8 n \tilde{F} \tilde{D}_{O} \tilde{C}_{O}^{*} \tilde{a}_{1} \tilde{a}_{2}}{\pi \tilde{L}}\left\{\operatorname{erfc}\left(\frac{\tilde{L}}{2 \sqrt{\tilde{D}_{R} \tilde{t}}}\right)+\frac{2}{\pi^{\frac{3}{2}}}\left(\frac{\tilde{a}_{1}}{\sqrt{\tilde{D}_{O} \tilde{t}}}+\frac{\tilde{a}_{2}}{\sqrt{\tilde{D}_{R} \tilde{t}}}\right) e^{-\frac{\tilde{L}^{2}}{4 \tilde{D}_{R} \tilde{t}}}\right\} .
$$

For an oxidation reaction, $\tilde{C}_{O}^{*}=0$ and $\tilde{C}_{R}^{*} \neq 0$, and the potential set-up will be the opposite. Then the current through the generator disk 1 is

$$
\tilde{I}_{1}(\tilde{t}) \approx 4 n \tilde{F} \tilde{D}_{R} \tilde{a}_{1} \tilde{C}_{R}^{*}\left\{1+\frac{2 \tilde{a}_{1}}{\pi^{\frac{3}{2}} \sqrt{\tilde{D}_{R} \tilde{t}}}+\frac{4 \tilde{a}_{1} \tilde{a}_{2}}{\pi^{2} \tilde{L}^{2}} \operatorname{erfc}\left(\frac{\tilde{L} \sqrt{\tilde{D_{O}}}+\tilde{L} \sqrt{\tilde{D}} \tilde{D}_{R}}{2 \sqrt{\tilde{D}_{O} \tilde{D}_{R} \tilde{t}}}\right)\right\},
$$

and the current through the collector disk 2 is:

$\tilde{I}_{2}(\tilde{t}) \approx-\frac{8 n \tilde{F} \tilde{D}_{R} \tilde{C}_{R}^{*} \tilde{a}_{1} \tilde{a}_{2}}{\pi \tilde{L}}\left\{\operatorname{erfc}\left(\frac{\tilde{L}}{2 \sqrt{\tilde{D}_{O} \tilde{t}}}\right)+\frac{2}{\pi^{\frac{3}{2}}}\left(\frac{\tilde{a}_{1}}{\sqrt{\tilde{D}_{R} \tilde{t}}}+\frac{\tilde{a}_{2}}{\sqrt{\tilde{D}_{O} \tilde{t}}}\right) e^{-\frac{\tilde{L}^{2}}{4 \tilde{D}_{O} \tilde{t}}}\right\}$.

Note the feedback effect in the generator currents, corresponding to the positive erfc terms in (69) and (71).

The steady-state collection efficiency is given in both cases by the simple formula:

$$
N=-\frac{\tilde{I}_{2}(\infty)}{\tilde{I}_{1}(\infty)}=\frac{2 \tilde{a}_{2}}{\pi \tilde{L}}+O\left(\epsilon^{3}\right)
$$


where $\epsilon=\tilde{a} / \tilde{L}$ and $\tilde{a}$ is the typical radius of the electrodes. This agrees with the first term in the series derived by Phillips and Stone [17], who also derived an $O\left(\epsilon^{3}\right)$ correction term, under the assumption that current density on the surface of the generator is uniform, which, although untrue, produces reasonable results. For generator-collector disks of unequal radii, the series derived by Phillips and Stone [17] is:

$$
N=\frac{2 \tilde{a}_{2}}{\pi \tilde{L}}+\frac{3 \tilde{a}_{1}^{2} \tilde{a}_{2}+4 \tilde{a}_{2}^{3}}{12 \pi \tilde{L}^{3}}+O\left(\epsilon^{5}\right) .
$$

For disks of equal radii, $\tilde{a}$, expression (73) becomes in non-dimensional terms:

$$
N=\frac{2 \epsilon}{\pi}+O\left(\epsilon^{3}\right),
$$

where $\epsilon=\tilde{a} / \tilde{L}$, and that of Phillips and Stone, (74), becomes:

$$
N=\frac{2 \epsilon}{\pi}+\frac{7 \epsilon^{3}}{12 \pi}+O\left(\epsilon^{5}\right)
$$

In Figure 3 (a), we plot $N$ as a function of the relative separation distance, $\tilde{L} / \tilde{a}=1 / \epsilon$. The solid black line represents our expression, (75), and the dashed line is plotted using the Phillips and Stone [17] expression (76) with their approximate $O\left(\epsilon^{3}\right)$ correction term. The line with circles is plotted using the steady-state limit of equation (10) in Cutress at al. [14], which was obtained by fitting to seven numerical simulations at different electrode separation distances up to $\tilde{L} / \tilde{a}=7$, and agreed well with previous simulations performed by Fulian et al. [11]. Figure 3 (b) shows the percentage differences between the formula of Cutress at al. and formulae (75) (solid line) and (76) (dashed line), calculated as $100 \times\left(N-N_{\text {Cutress }}\right) / N_{\text {Cutress. }}$. For $\tilde{L} / \tilde{a} \lesssim 2.6$, the Phillips and Stone formula (76) matches the Cutress values more closely than our formula, whilst for $\tilde{L} / \tilde{a} \gtrsim 2.6$ the reverse is true. The Phillips and Stone formula (76) matches the Cutress values to within $10 \%$ over the complete range of applicability of the Cutress formula, $2.1 \leq \tilde{L} / \tilde{a} \leq 7$. We remark that formulae (75) and (76) should converge to the Cutress formula as $\tilde{L} / \tilde{a}$ increases (and the asymptotic error in (75) and (76) tends to zero), but the range of applicability of the Cutress forumla is not large enough to test this; however we can observe that the difference is $O\left(\epsilon^{3}\right)$ at $\tilde{L} / \tilde{a}=7$ as expected. The advantages of formulae (75) and (76) are that they extend the Cutress formula to larger separation distances, and can be applied to disks of different radii by using the equivalent forms (73) and (74).

\subsection{Further discussion}

To illustrate the shielding and feedback effects encountered in a dual-disk system, we use the non-dimensional expression for the quasi-reversible current given by (59). For simplicity, we assume that there is only oxidant in the bulk, i.e. $C_{O}^{*}=1$ and $C_{R}^{*}=0$, and we take the diffusion coefficients of the oxidant and the reductant to be equal, $D_{O}=D_{R}=1$. We assume that the disks have equal radii, $a_{1}=a_{2}=1$, and that the relative separation of their centres is given by $1 / \epsilon=10$. We let disk 1 be polarised such that the current is near-diffusion-limited (or in generator mode) by 
setting $k_{f, 1}=10^{4}$ and $k_{b, 1}=10^{-4}$, and we vary the potential at disk 2 from neardiffusion-limited $\left(k_{f, 2}=10^{4}\right.$ and $\left.k_{b, 2}=10^{-4}\right)$, through quasi-reversible $\left(k_{f, 2}=1\right.$ and $k_{b, 2}=1$ ), to collector mode $\left(k_{f, 2}=10^{-4}\right.$ and $\left.k_{b, 2}=10^{4}\right)$. The results are shown in Figure 4. When disk 2 is in collector mode (triangles), the current at disk 1 (the generator) is enhanced due to feedback effects, and is greater than its current in isolation. When the potential at disk 2 is such that the reaction there is quasi-reversible (squares) or near-diffusion-limited (filled circles), both disks compete for the same species, and the current at disk 1 is reduced when compared to its current in isolation; this is the shielding effect, and it becomes greater as the potential at disk 2 approaches the diffusion-limited regime and competition between the electrodes increases. Figure 4 (c) indicates that the shielding effect can be substantial; although the separation distance is large (10 times the disk radii), the shielding effect approaches $6 \%$ as the current tends towards steady-state when both disks are in the near-diffusion-limited regime. In all cases, Figure 4 (c) shows that the shielding and feedback effects have more impact as time progresses, which is due to the increasing overlap of the diffusion layers of the two disks.

Lastly, we show that it can be important to account for differences in the diffusion coefficients of the oxidant and the reductant. In Figure 5, we plot the current responses at disk 1 for varying diffusion coefficients. The potentials at disk 1 and 2 are identical and are in the quasi-reversible regime, such that $k_{f, 1}=k_{b, 1}=k_{f, 2}=k_{b, 2}=1$. The remaining parameters are the same as above. For these parameters, the graphs in Figure 5 demonstrate that when the diffusion coefficient of the reductant is half that of the oxidant $\left(D_{O}=1\right.$ and $\left.D_{R}=0.5\right)$, the current is reduced by almost $25 \%$; similarly, when the diffusion coefficient of the reductant is double that of the oxidant $\left(D_{O}=1\right.$ and $D_{R}=2$ ), the current is increased by almost $20 \%$. Hence the impact of unequal diffusion coefficients can be significant.

\section{Conclusions}

We have derived an approximate asymptotic analytical expression for the timedependent chronoamperometric response of two inlaid disk electrodes. The expression is valid provided that the distance between the centres of the disks is much larger than their radii, and for long time-scales such that the diffusion layers of the electrodes interact. The analysis allows the electrodes to have different radii and to be held at different potentials; it also accommodates quasi-reversible reactions at the electrodes and different diffusion coefficients for the oxidant and the reductant. In particular, we have developed simple analytic expressions for the long-time-dependent current response and the steady-state collection efficiency of generator-collector systems. The analytical solution facilitates investigation of shielding and feedback effects in terms of the underlying parameters of the electrochemical system. Future work will be the extension of this analysis to arrays of inlaid disk electrodes, when understanding of the cross-talk between electrodes will be vital to predict the total current response. 


\section{References}

[1] E.O. Barnes, G.E.M. Lewis, S.E.C. Dale, F. Marken, R.G. Compton, Analyst 137 (2012) 1068-1081.

[2] K. Aoki, Electroanalysis 5 (1993) 627-639.

[3] C. Amatore, Electrochemistry at ultramicroelectrodes, in: I. Rubinstein (Ed.), Physical Electrochemistry: Principles, Methods and Applications, Marcel Dekker, New York, 1995, pp. 131-208.

[4] C.A. Basha, L. Rajendran, Int. J. Electrochem. Sci. 1 (2006) 268-282.

[5] A.M. Bond, K.B. Oldham, C.G. Zoski, J. Electroanal. Chem. 245 (1988) 71-104.

[6] F.-M. Matysik, U. Backofen, Fres. J. Anal. Chem. 356 (1996) 169-172.

[7] M. Zhong, J. Zhou, S.M. Lunte, G. Zhao, D.M. Giolando, J.R. Kirchhoff, Anal. Chem. 68 (1996) 203-207.

[8] C. Zhang, X. Zhou J. Electroanal. Chem. 415 (1996) 65-70.

[9] F.-M. Matysik, Electrochim. Acta 42 (1997) 3113-3116.

[10] J.E. Baur, H.M. Miller, M.A. Ritchason, Anal. Chim. Acta 397 (1999) 123-133.

[11] Q. Fulian, N.A. Williams, A.C. Fisher, Electrochem. Comm. 1 (1999) 124-127.

[12] F.L. Qiu, A.C. Fisher, Electrochem. Comm. 5 (2003) 87-93.

[13] J.E. Baur, P.N. Motsegood, J. Electroanal. Chem. 572 (2004) 29-40.

[14] I.J. Cutress, Y. Wang, J.G. Limon-Petersen, S.E.C. Dale, L. Rassaei, F. Marken, R.G. Compton, J.Electroanal. Chem. 655 (2011) 147-153.

[15] W. Strieder, J. Chem. Phys. 129 (2008) 134508.

[16] I.N. Sneddon, Mixed boundary value problems in potential theory, 1966, John Wiley \& Sons, Inc.

[17] C.G. Phillips, H.A. Stone, J. Electroanal. Chem. 437 (1997) 157-165.

[18] C.G. Bell, P.D. Howell, H.A. Stone, W.-J. Lim, J.H. Siggers, J. Electroanal. Chem. 673 (2012) 48-57.

[19] J. Newman, The Fundamental Principles of Current Distribution and Mass Transport in Electrochemical Cells, in: A. J. Bard (Ed.), Electroanalytical Chemistry, Vol. 6, Marcel Dekker Inc., New York, 1973, pp. 187-352.

[20] D.R. Baker, M.W. Verbrugge, J. Electrochem. Soc. 137 (1990) 1832-1842. 
[21] M. Abramowitz, I.A. Stegun, (Eds.) Handbook of Mathematical Functions with Formulas, Graphs, and Mathematical Tables, National Bureau of Standards Applied Mathematics Series, 55, U.S. Government Printing Office, Washington, DC, 1964.

[22] C.J. Tranter, Quart. J. Mech. and Appl. Math. 4 (1950) 411-419.

[23] C.G. Phillips, J. Electroanal. Chem. 291 (1990) 251-256.

[24] E.J. Hinch, Perturbation Methods, first ed., Cambridge University Press, Cambridge, 1991. 


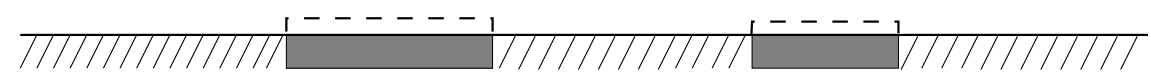

(a) Diffusion layer at short times

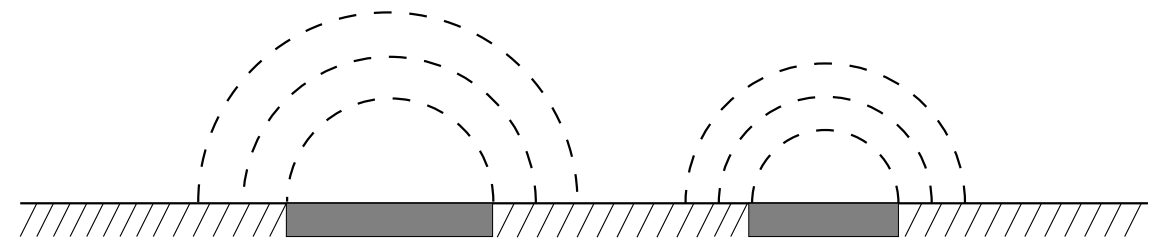

(b) Diffusion layer at intermediate times

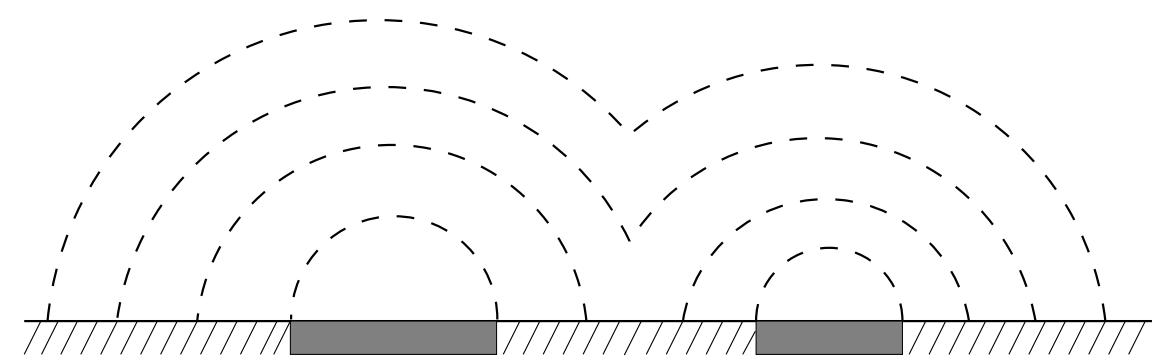

(c) Diffusion layer at longer times

Figure 1: Cartoon depicting the evolution of the diffusion layers (indicated by dashed lines) at neighbouring disk electrodes (shaded grey) of different sizes. (a) At short times, the disks are independent and the diffusion is effectively planar. (b) At intermediate times, the disks remain independent but the diffusion layers have grown to be three-dimensional. (c) Finally, over time-scales such that the species have had time to diffuse between the electrodes, the diffusion layers interact and impact the resultant current through each electrode. This is the stage at which the analytical solutions derived in this article are valid. 
Far-field, $\tilde{r}^{2}+\tilde{z}^{2} \rightarrow \infty$ $\tilde{C}_{O} \rightarrow \tilde{C}_{O}^{*}, \quad \tilde{C}_{R} \rightarrow \tilde{C}_{R}^{*}$
Governing equations, $\tilde{z}>0$

$$
\begin{aligned}
& \tilde{D}_{O} \nabla^{2} \tilde{C}_{O}=\frac{\partial \tilde{C}_{O}}{\partial \tilde{t}} \\
& \tilde{D}_{R} \nabla^{2} \tilde{C}_{R}=\frac{\partial \tilde{C}_{R}}{\partial \tilde{t}}
\end{aligned}
$$

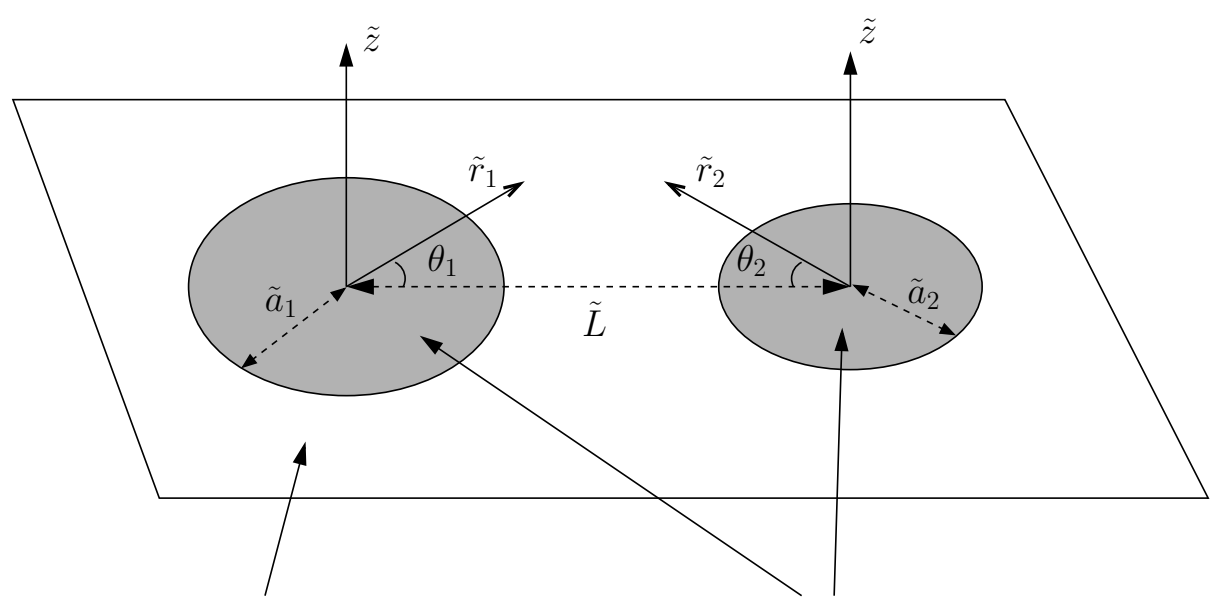

Insulator, $\tilde{r}_{j}>\tilde{a}_{j}, \tilde{z}=0$

Electrodes, $\tilde{r}_{j} \leq \tilde{a}_{j}, \tilde{z}=0$

$$
\tilde{D}_{O} \frac{\partial \tilde{C}_{O}}{\partial \tilde{z}}=\tilde{D}_{R} \frac{\partial \tilde{C}_{R}}{\partial \tilde{z}}=0
$$$$
\tilde{D}_{O} \frac{\partial \tilde{C}_{O}}{\partial \tilde{z}}=\tilde{k}_{f, j} \tilde{C}_{O}-\tilde{k}_{b, j} \tilde{C}_{R}
$$$$
\tilde{D}_{O} \frac{\partial \tilde{C}_{O}}{\partial \tilde{z}}=-\tilde{D}_{R} \frac{\partial \tilde{C}_{R}}{\partial \tilde{z}}
$$

Figure 2: Schematic depicting the theoretical problem addressed in this article. An oxidant and a reductant diffuse with constant diffusion coefficients, $\tilde{D}_{O}$ and $\tilde{D}_{R}\left(\mathrm{~m}^{2} \mathrm{~s}^{-1}\right)$, above two circular disk electrodes inlaid into an insulating plane at $\tilde{z}=0$. The concentrations of the species are denoted by $\tilde{C}_{O}(\tilde{x}, \tilde{y}, \tilde{z}, \tilde{t})$ and $\tilde{C}_{R}(\tilde{x}, \tilde{y}, \tilde{z}, \tilde{t})\left(\mathrm{mol} \mathrm{m}^{-3}\right)$ respectively, and their bulk concentrations, $\tilde{C}_{O}^{*}$ and $\tilde{C}_{R}^{*}\left(\mathrm{~mol} \mathrm{~m}^{-3}\right)$, are constant in the far-field. The disk electrodes have radii $\tilde{a}_{1,2}(\mathrm{~m})$ and their centres are separated by a distance $\tilde{L}(\mathrm{~m})$. At each electrode $j=1,2$, the two species exchange $n$ electrons according to a standard redox reaction (3) with constant forward and backward reaction rates denoted by $\tilde{k}_{f, j}$ and $\tilde{k}_{b, j}\left(\mathrm{~m} \mathrm{~s}{ }^{-1}\right)$. For the purposes of our analysis it is convenient to introduce two sets of cylindrical polar coordinates $\left(\tilde{r}_{j}, \tilde{z}, \theta_{j}\right)$, with origins at the centre of each electrode and $\theta_{j}$ measured from the line joining the centres as shown in the diagram. 


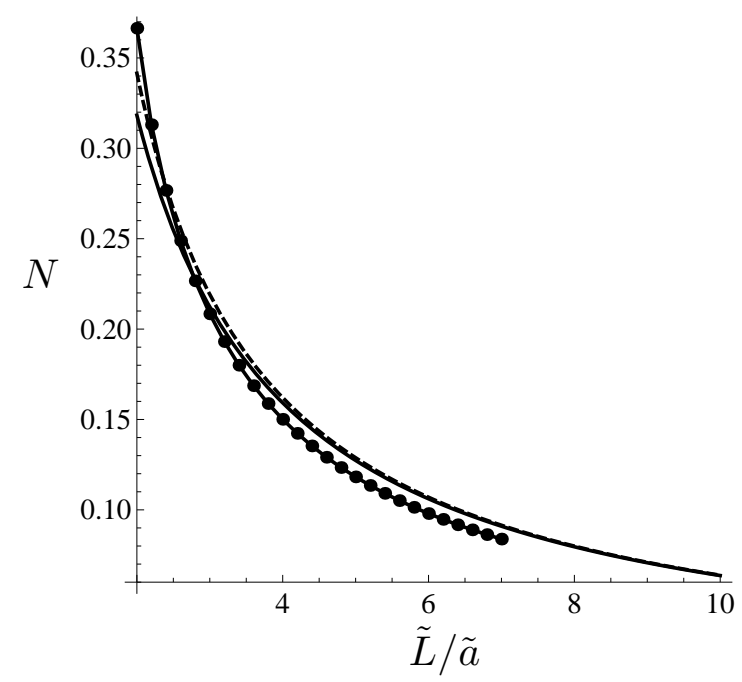

(a) Collection efficiency, $N$

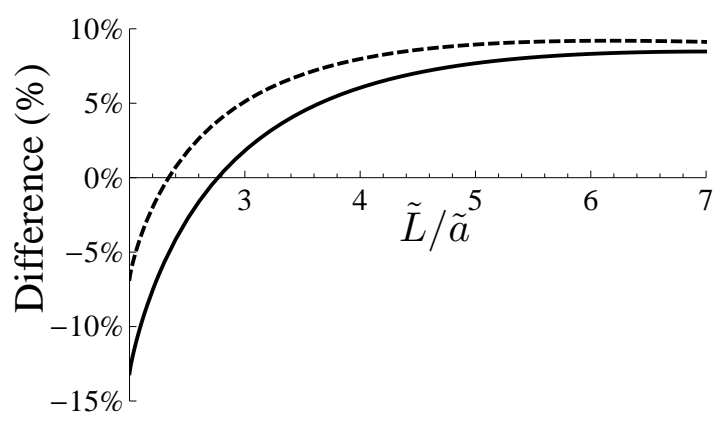

(b) Difference $(\%)$

Figure 3: Plot (a) shows the collection efficiency, $N$, of two disk electrodes with equal radii, $\tilde{a}$, in generatorcollector mode as a function of the non-dimensional centre-to-centre separation distance $\tilde{L} / \tilde{a}$. The solid line represents the analytical formula (75), whilst the dashed line represents the formula (76) derived by Phillips and Stone [17] with the approximate $O\left((\tilde{a} / \tilde{L})^{3}\right)$ correction term. The line with circles is the steady-state limit of the formula derived by Cutress et al. [14] by fitting to numerical simulations for $2.1 \leq \tilde{L} / \tilde{a} \leq 7$ (expression (10) in their article). Plot (b) shows the percentage difference between the Cutress formula and formulae (75) (solid line) and (76) (dashed line). For $\tilde{L} / \tilde{a} \lesssim 2.6$, the Phillips and Stone formula (76) matches the Cutress values more closely than our formula (75), whilst for $\tilde{L} / \tilde{a} \gtrsim 2.6$ the reverse is true. The Phillips and Stone formula (76) matches the Cutress formula to within $10 \%$ over the range of its applicability $2.1 \leq \tilde{L} / \tilde{a} \leq 7$. 


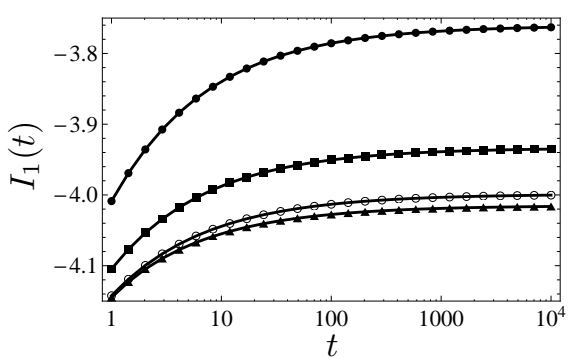

(a) $I_{1}(t)$

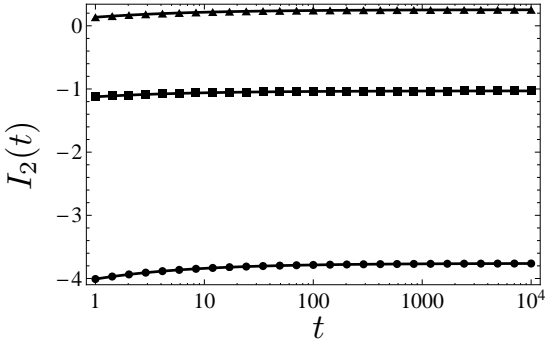

(b) $I_{2}(t)$

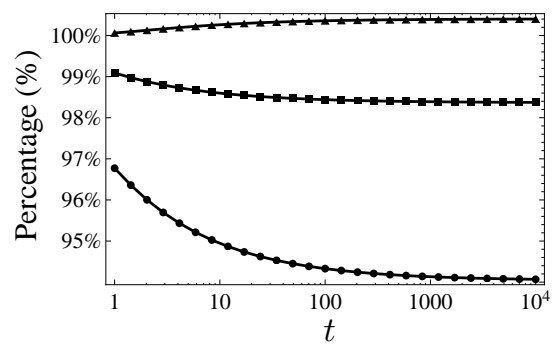

(c) $I_{1}(t) /\left\{\left.I_{1}(t)\right|_{a_{2}=0}\right\}(\%)$

Figure 4: Figures (a) and (b) show log-linear plots of the non-dimensional time-dependent currents through disks 1 and $2, I_{1}(t)$ and $I_{2}(t)$, calculated from formula (59). The disks have equal radii, $a_{1}=a_{2}=1$, and their centre-to-centre separation is given by $1 / \epsilon=10$. The bulk concentrations of oxidant and reductant are $C_{O}^{*}=1$ and $C_{R}^{*}=0$, and their diffusion coefficients are equal, $D_{O}=D_{R}=1$. The potential at disk 1 is held in the near-diffusion-limited regime (or generator mode) by setting $k_{f, 1}=10^{4}$ and $k_{b, 1}=10^{-4}$. The different curves in Figures (a) and (b) correspond to varying the potential at disk 2: filled circles are neardiffusion-limited, $k_{f, 2}=10^{4}$ and $k_{b, 2}=10^{-4}$; squares are quasi-reversible, $k_{f, 2}=1$ and $k_{b, 2}=1$; and, triangles are collector mode, $k_{f, 2}=10^{-4}$ and $k_{b, 2}=10^{4}$. The extra curve in Figure (a) with unfilled circles is the near-diffusion-limited current at disk $1\left(k_{f, 1}=10^{4}\right.$ and $\left.k_{b, 1}=10^{-4}\right)$ in the absence of disk $2,\left.I_{1}(t)\right|_{a_{2}=0}$, obtained by setting $a_{2}=0$ in (59). Figure (c) shows the time-dependent current through disk 1 as a percentage of its value in isolation to illustrate the impact of adding disk 2, which results in shielding and feedback effects. Note that the shielding effect can be substantial; although the separation distance is large (10 times the radii), the shielding effect approaches $6 \%$ as the current tends towards steady-state when both disks are in the near-diffusion-limited regime (filled circles). 


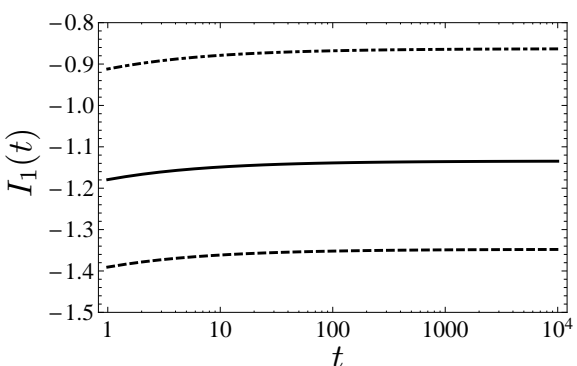

(a) $I_{1}(t)$

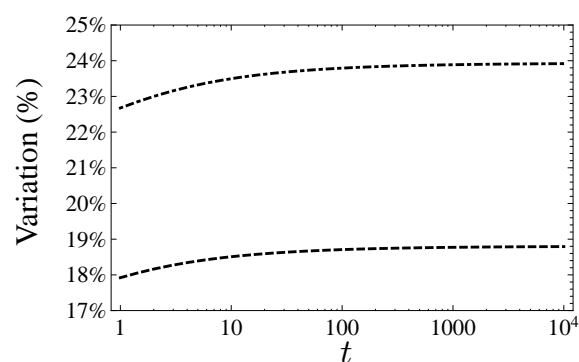

(b) Absolute percentage variation

Figure 5: Illustration of the substantial impact of unequal diffusion coefficients on the current response. Figure (a) shows the non-dimensional current responses through disk $1, I_{1}(t)$, calculated from formula (59), when the diffusion coefficients are varied: solid line, $D_{O}=1$ and $D_{R}=1$; dashed line, $D_{O}=1$ and $D_{R}=2$; and, dot-dashed line, $D_{O}=1$ and $D_{R}=0.5$. The potentials at both electrodes are identical: $k_{f, 1}=k_{b, 1}=k_{f, 2}=k_{b, 2}=1$. Other parameters are $C_{O}^{*}=1, C_{R}^{*}=0, a_{1}=a_{2}=1$, and $\epsilon=0.1$. By symmetry, the chosen parameters imply that the currents through disks 1 and 2 are identical; hence we only display the current through disk 1. Figure (b) shows the large time-dependent absolute percentage variations from the equal-diffusion-coefficient current response when the diffusion coefficients are unequal. 